11. Shiloh, Y. ATM and ATR: networking cellular responses to DNA damage. Curr. Opin. Gen. Dev. 11 71-77 (2001).

12. Kim, S. T., Lim, D. S., Canman, C. E. \& Kastan, M. B. Substrate specificities and identification of putative substrates of ATM kinase family members. J. Biol. Chem. 274, 37538-37543 (1999).

13. Tibbetts, R. S. et al. A role for ATR in the DNA damage-induced phosphorylation of p53. Genes Dev. 13, 152-157 (1999).

14. Banin, S. et al. Enhanced phosphorylation of p53 by ATM in response to DNA damage. Science 281, 1674-1677 (1998)

15. Cliby, W. A. et al. Overexpression of a kinase-inactive ATR protein causes sensitivity to DNAdamaging agents and defects in cell cycle checkpoints. EMBO J. 17, 159-169 (1998).

16. Tibbetts, R. S. et al. Functional interactions between BRCAl and the checkpoint kinase ATR during genotoxic stress. Genes Dev. 14, 2989-3002 (2000).

17. Burtelow, M. A., Kaufmann, S. H. \& Karnitz, L. M. Retention of the human Rad9 checkpoint complex in extraction-resistant nuclear complexes after DNA damage. J. Biol. Chem. 275, 26343-26348 (2000)

18. Sekulic, A. et al. A direct linkage between the phosphoinositide 3-kinase-AKT signaling pathway and the mammalian target of rapamycin in mitogen-stimulated and transformed cells. Cancer Res. 60,
3504-3513 (2000).

19. He, T. C. et al. A simplified system for generating recombinant adenoviruses. Proc. Natl Acad. Sci. USA 95, 2509-2514 (1998).

\section{Acknowledgements}

We thank B. Vogelstein for the pAdTrack-CMV and pAdEasy adenoviral vectors; Y. Xu for the $A T M^{+/+}$and $A T M^{-1-}$ mouse embryo fibroblasts; C. Counter for the BJ fibroblasts; L. M. Karnitz for the anti-hRad17 antibody; L. Martinek and M. Cook for the flow cytometric analysis; Y. Yu for technical help; and members of the Wang and Abraham laboratories for scientific discussions. This work was supported by grants from the National Institutes of Health, the A-T Children's Project, and the Johnson and Johnson Foundation.

Correspondence and requests for materials should be addressed to X.-F.W (e-mail: wang0011@mc.duke.edu).

\section{corrections}

\section{Gain-assisted superluminal light propagation}

\section{J. Wang, A. Kuzmich \& A. Dogariu}

Nature 406, 277-279 (2000).

It has been drawn to our attention that we inadvertently did not cite a previous paper by Segard and $\mathrm{Macke}^{1}$ on the topic of observed superluminal light pulse propagation in media with heavy absorption using a direct pulse shape detection technique. We did, however, cite an earlier paper by Chu and $\mathrm{Wong}^{2}$ on this topic using a different technique. Our introductory statement "in all previous experimental demonstrations, the light pulses experienced either very large absorption or severe reshaping" stands. We have now included this reference in a more detailed description ${ }^{3}$ of our experiment.

1. Segard, B. \& Macke, B. Observation of negative velocity pulse propagation. Phys. Lett. A 109, 213-216 (1985).

2. Chu, S. \& Wong, S. Linear pulse propagation in an absorbing medium. Phys. Rev. Lett. 48, 738-741 (1982).

3. Dogariu, A., Kuzmich, A. \& Wang, L. J. Transparent anomalous dispersion and superluminal light pulse propagation at a negative group velocity. Phys. Rev. A 63, 053806 (2001).

\section{Endogenous cannabinoids mediate retrograde signalling at hippocampal synapses}

\section{R. I. Wilson \& R. A. Nicoll}

Nature 410, 588-592 (2001).

It has come to our attention that we failed to cite a relevant study (Egertová, M., Giang, D. K., Cravatt, B. F. \& Elphick, M. R. Proc. R. Soc. Lond. B 265, 2081-2085; 1998) in our Letter. Based on the immunolocalization of fatty acid amide hydrolase, which inactivates endocannabinoids, to hippocampal pyramidal cells, and the localization of the $\mathrm{CB} 1$ receptor to presynaptic terminals, Egertová et al. proposed that endocannabinoids might function as retrograde messengers.

\title{
Genomic analysis of metastasis reveals an essential role for RhoC
}

\section{E. A. Clark, T. R. Golub, E. S. Lander \& R. 0. Hynes}

Nature 406, 532-535 (2000).

A citation of previous work by del Paso et al. ${ }^{1}$ as evidence in support of our results was erroneously removed during editing of an earlier draft.

1. del Paso, L., Hernandez-Alcoceba, R., Embade, N., Carnero, A., Esteve, P., Paje, C. \& Lacal, J. C.

Oncogene 15, 3047-3057 (1997). 


\section{Gain-assisted supertuminal light propagation}

\section{J. Wang, A. Kuzmich \& A. Dogariu}

NEC Research Institute, 4 Independence Way, Princeton, New Jersey 08540, USA

Einstein's theory of special relativity and the principle of causality $^{1-4}$ imply that the speed of any moving object cannot exceed that of light in a vacuum $(c)$. Nevertheless, there exist various proposals $^{5-18}$ for observing faster-than-c propagation of light pulses, using anomalous dispersion near an absorption line ${ }^{4,6-8}$, nonlinear ${ }^{9}$ and linear gain lines ${ }^{10-18}$, or tunnelling barriers ${ }^{19}$. However, in all previous experimental demonstrations, the light pulses experienced either very large absorption ${ }^{7}$ or severe reshaping ${ }^{9,19}$, resulting in controversies over the interpretation. Here we use gain-assisted linear anomalous dispersion to demonstrate superluminal light propagation in atomic caesium gas. The group velocity of a laser pulse in this region exceeds $c$ and can even become negative ${ }^{16,17}$, while the shape of the pulse is preserved. We measure a group-velocity index of $n_{\mathrm{g}}=-310( \pm 5)$; in practice, this means that a light pulse propagating through the atomic vapour cell appears at the exit side so much earlier than if it had propagated the same distance in a vacuum that the peak of the pulse appears to leave the cell before entering it. The observed superluminal light pulse propagation is not at odds with causality, being a direct consequence of classical interference between its different frequency components in an anomalous dispersion region.

When a light pulse of frequency $v$ and bandwidth $\Delta v$ enters a dispersive linear medium of an optical refractive index $n(v)$, the light pulse propagates at the group velocity $v_{\mathrm{g}}=c / n_{\mathrm{g}}$, where

a

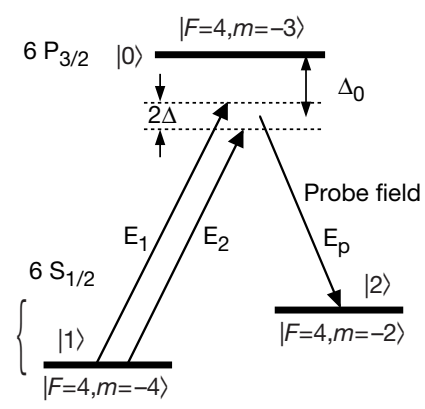

b

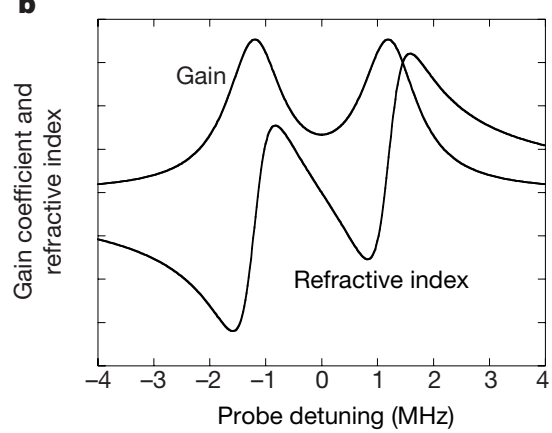

Figure 1 Gain-assisted anomalous dispersion. a, Schematic atomic level diagram. b. Frequency-dependent gain coefficient and refractive index obtained from equation (1) for a probe light beam propagating through an atomic medium with its level structure shown in $\mathbf{a}$ $n_{\mathrm{g}}=n(v)+v \mathrm{~d} n(v) / \mathrm{d} v$ is the group-velocity index. If the groupvelocity index remains constant over the pulse bandwidth $\Delta v$, the light pulse maintains its shape during propagation. In recent experiments involving electromagnetically induced transparency $(\mathrm{EIT})^{20-22}$, the group-velocity index was greatly enhanced using the lossless normal dispersion region between two closely spaced absorption lines. Thus the group velocity of light was dramatically reduced to as slow as $8 \mathrm{~m} \mathrm{~s}^{-1}$ (refs 23-25). Conversely, between two closely spaced gain lines ${ }^{16}$, an anomalous dispersion region appears where $v \mathrm{~d} n(v) / \mathrm{d} v$ is negative and its magnitude can become large. In this situation, the group velocity of a light pulse can exceed $c$ and can even become negative ${ }^{16,17}$.

A negative group velocity of light is counterintuitive but can be understood as follows. For a medium of a length $L$, it takes a propagation time $L / v_{\mathrm{g}}=n_{\mathrm{g}} L / c$ for a light pulse to traverse it. Compared with the propagation time for light to traverse the same distance in a vacuum, that is, the vacuum transit time $L / c$, the light pulse that enters the medium will exit at a moment that is delayed by a time difference $\Delta T=L / v_{\mathrm{g}}-L / c=\left(n_{\mathrm{g}}-1\right) L / c$. When $n_{\mathrm{g}}<1$, the delay time $\Delta T$ is negative, resulting in an advancement. In other words, when incident on a medium with group-velocity index $n_{\mathrm{g}}<1$, a light pulse can appear on the other side sooner than if it had traversed the same distance in a vacuum ${ }^{5,15}$. Furthermore, in contradiction to traditional views that a negative group velocity of light has no physical meaning, when the group-velocity index becomes negative, the pulse advancement $-\Delta T=\left(1-n_{\mathrm{g}}\right) L / c$ becomes larger than the vacuum transit time $L / c$. In other words, it appears as if the pulse is leaving the cell even before it enters. This counterintuitive phenomenon is a consequence of the wave nature of light.

The principle of the experimental realization of a lossless anomalous dispersion region and hence gain-assisted superluminality (GAS) is illustrated in Fig. 1a. In a gaseous medium of atoms each of which has three levels: an excited state $|0\rangle$ and two ground states $|1\rangle$ and $|2\rangle$, we first prepare all atoms to be in a ground state $|1\rangle$ by optical pumping. For simplicity, we first ignore the Doppler shift and assume that the atoms are at rest. We apply two strong continuous-wave (CW) Raman pump light beams $\mathrm{E}_{1}$ and $\mathrm{E}_{2}$ that propagate through the atomic medium. The frequencies of $\mathrm{E}_{1}$ and $\mathrm{E}_{2}, v_{1}$ and $v_{2}$, are different by a small amount $2 \Delta$ and both fields are detuned from the atomic transition frequency $v_{01}(|0\rangle$ to $|1\rangle)$ by a large average amount $\Delta_{0}$. Since the Rabi frequencies associated with the fields $\mathrm{E}_{1}$ and $\mathrm{E}_{2}$ are small compared with the common detuning $\Delta_{0}$, the atoms mostly remain in state $|1\rangle$. When a probe light beam $\mathrm{E}_{\mathrm{p}}$ is introduced, a Raman transition can occur, causing an atom to absorb a Raman pump photon from the fields $\mathrm{E}_{1}$ or $\mathrm{E}_{2}$ and emit a photon into the field $E_{p}$ while making a transition from $|1\rangle$ to $|2\rangle$. Obviously, there are two frequencies where the gain in the probe field is maximized. The maximum gain occurs when the probe field is resonant with the Raman transitions caused by either of the two pump fields $E_{1}$ and $E_{2}$. The optical susceptibility of the probe field can thus be derived as

$$
\chi(v)=\frac{M_{1}}{v-v_{1}+i \gamma}+\frac{M_{2}}{v-v_{2}+i \gamma}
$$

Here $M_{1,2}=\frac{\left|\mu_{02}\right|^{2}}{4 \pi \hbar \epsilon_{0}} \frac{\left|\Omega_{1,2}\right|^{2}}{\Delta \Delta_{0}^{2}} N$ with $\mu_{02}, \Omega_{1,2}$ and $N$ being the dipole moment of the $|0\rangle$ to $|2\rangle$ atomic transition, the Rabi frequencies of the Raman pump fields $\mathrm{E}_{1}$ and $\mathrm{E}_{2}$ and the effective atomic density difference of states $|1\rangle$ and $|2\rangle$, respectively. $\gamma$ is the Raman transition inverse lifetime. The refractive index and the gain coefficient obtained using the susceptibility given in equation (1) are shown in Fig. 1b. In the region between the two gain lines, an anomalous dispersion region appears.

However, in a gaseous atomic medium, there is Doppler broadening: for atoms moving at different velocities in the light propagation direction, the common detuning $\Delta_{0}$ is shifted. The effects of Doppler broadening are twofold. First, the $M$-factors in equation 
(1) are replaced by

$$
M_{1,2}=\frac{\left|\mu_{02}\right|^{2}}{4 \pi \hbar \epsilon_{0}} \int \mathrm{d} V \frac{\left|\Omega_{1,2}\right|^{2}}{\left(\Delta_{0}+v V / c\right)^{2}} N(V)
$$

Here $N(V)$ is the effective density of atoms in the velocity group $V$. The common detuning $\Delta_{0}$ is replaced by the Doppler detuning $\Delta_{0}+v V / c$. However, the quadratic dependence of the coefficient $M$ on the Doppler detuning prevents cancellation. Second, inside the Doppler profile, the expression for $M$ given above appears to become singular when the detuning $\Delta_{0}+v V / c$ vanishes. This is automatically avoided in practice because for atoms with detuning $\Delta_{0}+v V / c$ that is less than a certain linewidth containing contributions from the natural linewidth and power broadening, the Raman pump beams act like reversed optical pumping beams that deplete these velocity groups. For atoms in the velocity group where the effective detuning $\Delta_{0}+v V / c$ vanishes, $N(V)$, the atom number difference also vanishes. Finally, the atoms that are pumped away from the level $|1\rangle$ act as a weak broadband absorber. This compensates for the small residual gain in the region between the two Raman gain lines shown in Fig. $1 \mathrm{~b}$.

The experiment was performed using an atomic caesium (Cs) vapour cell at $30^{\circ} \mathrm{C}$ and the main set-up is shown in Fig. 2. The caesium atoms are confined in a 6-cm-long Pyrex glass cell coated with paraffin for the purpose of maintaining ground-state spin polarization. The atomic cell is placed in a small $(1.0 \mathrm{G})$ uniform magnetic field parallel to the light propagation direction. In region I, two laser beams optically pump the atoms into the ground-state hyperfine magnetic sub-level $|F=4, m=-4\rangle$ that serves as state $|1\rangle$ (Fig. 1a). One left-hand $(\sigma-)$ polarized laser beam from a narrow linewidth diode laser is tuned to the $852-\mathrm{nm} \mathrm{D}_{2}$ transitions to empty the $6 \mathrm{~S}_{1 / 2} F=3$ hyperfine ground state. We further apply a second laser $(\sigma-)$ to optically pump the atoms into the $|F=4, m=-4\rangle$ state via the $\mathrm{D}_{1}$ transitions to the $6 \mathrm{P}_{1 / 2}$ hyperfine excited states. When atoms collide with the paraffin-coated glass walls, they change their velocities inside the Doppler profile while remaining in the ground state $|F=4, m=-4\rangle$ and hence the majority of the caesium atoms inside the cell are prepared into this state. In region II, three light beams derived from the same laser propagate colinearly through the cell. Two strong CW Raman pump beams are right-hand circularly polarized $(\sigma+)$ and are frequency-shifted by

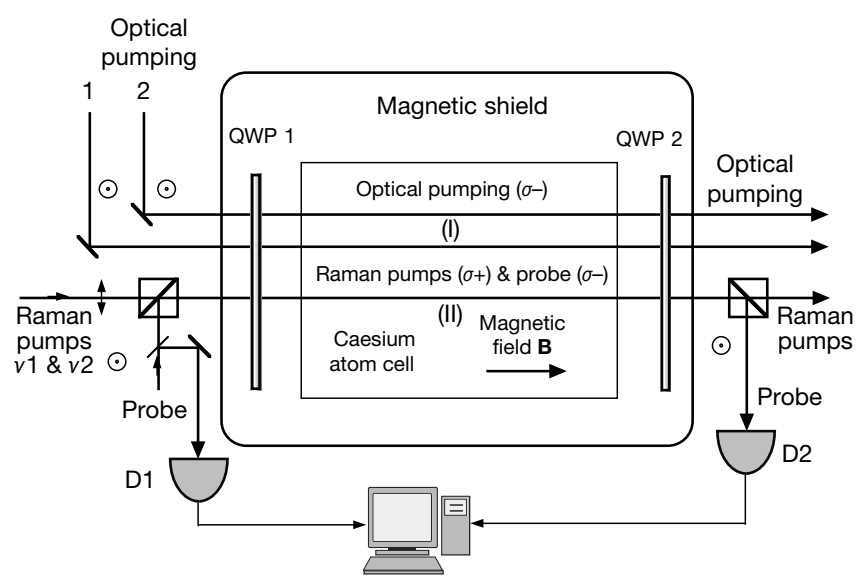

Figure 2 Schematic experimental set-up. Two optical pumping beams tuned to the caesium $(C s)$ atomic $D_{1}$ and $D_{2}$ transitions prepare the atoms in its ground-state hyperfine sublevel $|F=4, m=4\rangle$. Two Raman pump beams and a Raman probe beam derived from a common narrow linewidth diode laser propagate co-linearly parallel to a small magnetic field $\mathbf{B}$ through the atomic cell. Two $\lambda / 4$ plates (QWP1\&2) are used to prepare the three light beams into the corresponding circular polarization states and then separate them for analysis.
2.7 $\mathrm{MHz}$ using two acousto-optical (A/O) modulators. The linewidths of the A/O modulators are $20 \mathrm{kHz}$. A third light beam, the probe beam, is left-hand polarized $(\sigma-)$ and by using another A/O can be tuned in frequency and operate either in CW or pulsed mode. In the experiment, the pre-emptied hyperfine magnetic sublevel $|F=4, m=-2\rangle$ serves as the Raman transition final state $|2\rangle$. The intermediate Raman transitional state $|0\rangle$ is served primarily by the hyperfine sublevel $|F=4, m=-3\rangle$ of the $6 \mathrm{P}_{3 / 2}$ excited state with additional contributions from transitions through $|F=3, m=-3\rangle$ and $|F=5, m=-3\rangle$ hyperfine sublevels.

First, we operate the Raman probe beam in a tunable CW mode to measure the gain and refractive index of the atomic system as a function of the probe frequency detuning. Figure 3 shows the measured gain coefficient and the refractive index. In order to obtain the gain coefficient, we first measure the intensity of the transmitted probe beam as a function of probe frequency. We then extract the gain coefficient. The refractive index is measured using a radio-frequency interferometric technique. The superimposed curve is obtained from equation (1) using parameters obtained from the gain measurement. From Fig. 3, we see that a negative change of $\Delta n=-1.8 \times 10^{-6}$ in the index occurs over a narrow probe frequency range of $\Delta v=1.9 \mathrm{MHz}$ between the two gain lines. Using the expression of the group-velocity index, we obtain the result $n_{\mathrm{g}}=-330( \pm 30)$ in that frequency region. The $10 \%$ error reflects the accuracy of the phase measurement.

Next, a pulsed Raman probe beam is employed to observe the superluminal propagation. A near-gaussian probe pulse with a 3.7$\mu$ s full-width at half-maximum (FWHM) is generated by applying an electronic pulse to the probe beam $\mathrm{A} / \mathrm{O}$ modulator. A portion of the pulsed probe beam is divided at a beam-splitter before the atomic cell and aligned onto photodiode D1 as a reference. Because the total number of atoms in the probe volume limits the maximum net energy gain of the probe pulse, we use a very weak probe beam $(<1 \mu \mathrm{W})$ in order to avoid saturation and hence to optimize the anomalous dispersion. A high-sensitivity avalanche photodiode, reverse-biased below breakdown, serves as detector D2 to measure the weak probe pulse that propagates through the atomic cell. The photoelectric current produced by detector D2 is converted to a voltage signal using a $500-\Omega$ load resistor and recorded by a digitizing oscilloscope using a synchronized output signal from the pulse generator as the trigger. Pulses from detector D1 are also recorded.

In order to measure the pulse propagation time, we first tune the diode laser that produces the Raman pump and probe beams far offresonance from the $852-\mathrm{nm}$ caesium $\mathrm{D}_{2}$ lines (by $2.5 \mathrm{GHz}$ ) to

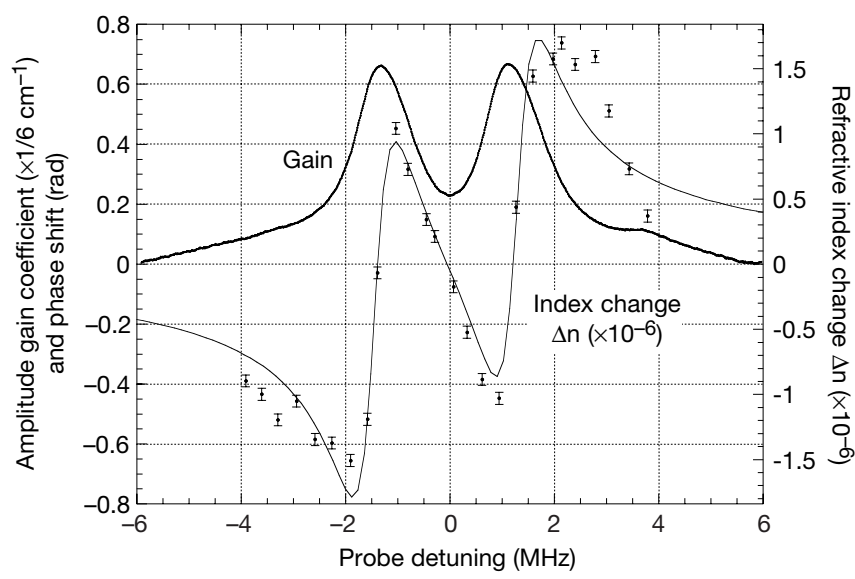

Figure 3 Measured refractive index and gain coefficient. The superimposed curve over the index data is obtained using equation (1) with parameters $v_{1}, v_{2}$ and $\gamma$ obtained experimentally. 
measure the time-dependent probe-pulse intensity. When the laser is placed far off-resonance, the atoms have no effect and the probe pulse propagates at speed $c$ inside the cell. We then tune the diode laser back to within the Doppler absorption profile and lock the laser on its side. Using the same synchronized pulse generator output signal as the trigger, we record the time-dependent probepulse intensity measured by detector D2. We verify that no systematic drift is present by tuning the laser off-resonance again by the same amount and record the probe-pulse signal; the two offresonance pulses are identical to within less than 1 ns. Probe pulses both on and off-resonance are shown in Fig. 4. Probe pulses on resonance show a $40 \%$ transmittance and this is due to the broadband absorption of those atoms reverse-pumped away from the $|F=4, m=-4\rangle$ state. It is evident that there is almost no change in the pulse shape. The front edges and the trailing edges of the pulses are shown in the insets; both edges are shifted forward by the same amount. Using a least-squares fitting procedure, we obtain a pulse advancement shift of $62( \pm 1) \mathrm{ns}$. Compared with the $0.2-\mathrm{ns}$ propagation time for light to traverse the $6-\mathrm{cm}$ length of the atomic cell in vacuum, the 62-ns advancement gives an effective groupvelocity index of $n_{\mathrm{g}}=-310( \pm 5)$. The small discrepancy with the group-velocity index inferred from the refractive index data is due to experimental errors. The pulses measured with detector D1 are also recorded in the sequence of the off-, on-, off-resonance pulsepropagation measurements and are found to be identical to within $1.5 \mathrm{~ns}$.

Here we note that the physical mechanism that governs the observed superluminal light propagation differs for the previously studied anomalous dispersion associated with an absorption or a gain resonance ${ }^{5-17}$. Specifically, in the anomalous dispersion region of a single gain resonance, the superluminal propagation of a pulse has been viewed as the result of the amplification of the pulse front edge and absorption of its tail ${ }^{5}$. In the present experiment, the 3.7- $\mu$ s FWHM probe pulse has only a $120-\mathrm{kHz}$ bandwidth (FWHM) that is much narrower than the $2.7-\mathrm{MHz}$ separation of the two gain lines and the probe pulse is placed in the middle of these gain lines spectrally. The probe pulse thus contains essentially no spectral components that are resonant with the Raman gain lines to be amplified. Therefore, the argument that the probe pulse is advanced by amplification of its front edge does not apply. The superluminal light propagation observed here is the result only of the anomalous

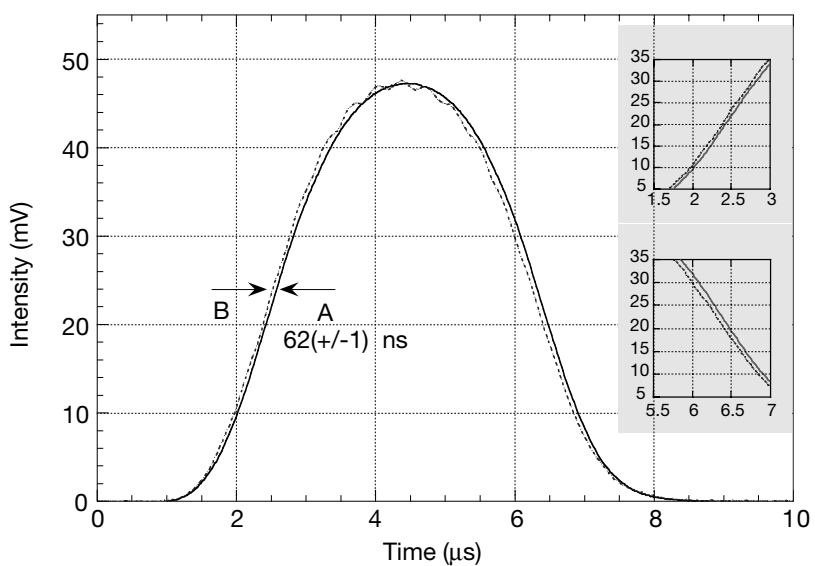

Figure 4 Measured pulse advancement for a light pulse traversing through the caesium vapour in the gain-assisted superluminality state. A indicates a light pulse far offresonance from the caesium $D_{2}$ transitions propagating at speed $c$ through $6 \mathrm{~cm}$ of vacuum. $B$ shows the same light pulse propagating through the same caesium-cell near resonance with a negative group velocity $-c / 310$. Insets show the front and trailing portions of the pulses. Pulses $A$ and $B$ are both the average of 1,000 pulses. The offresonance pulse $(A)$ is normalized to the magnitude of $B$. dispersion region created with the assistance of two nearby Raman gain resonances. We emphasize that the observed superluminal light propagation is a result of the wave nature of light ${ }^{2}$. It can be understood by the classical theory of wave propagation in an anomalous dispersion region where interference between different frequency components produces this rather counterintuitive effect.

Finally, we note that the observed superluminal light pulse propagation is not at odds with causality or special relativity. In fact, the very existence of the lossless anomalous dispersion region given in equation (1) is a result of the Kramers-Kronig relation which itself is based on the causality requirements of electromagnetic responses ${ }^{3,5}$. Remarkably, the signal velocity ${ }^{4}$ of a light pulse, defined as the velocity at which the half point of the pulse front travels, also exceeds the speed of light in a vacuum, $c$, in the present experiment. It has also been suggested ${ }^{4,16}$ that the true speed at which information is carried by a light pulse should be defined as the "frontal" velocity of a step-function-shaped signal which has been shown not to exceed $c$ (ref. 4). The implications of the present experiment on signal propagation and its speed will be further analysed, particularly for the case when the light pulse consists of only a few photons.

Received 11 May; accepted 26 June 2000.

1. Einstein, A., Lorentz, H. A., Minkowski, H. \& Weyl, H. The Principle of Relativity, Collected Papers (Dover, New York, 1952).

2. Born, M. \& Wolf, E. Principle of Optics 7th edn (Cambridge Univ. Press, Cambridge, 1997).

3. Landau, L. D. \& Lifshitz, E. M. Electrodynamics of Continuous Media (Pergamon, Oxford, 1960).

4. Brillouin, L. Wave Propagation and Group Velocity (Academic, New York, 1960).

5. Chiao, R. Y. in Amazing Light, a Volume Dedicated to C. H. Townes on His Eightieth Birthday (ed. Chiao, R. Y.) 91-108 (Springer, New York, 1996).

6. Garrett, C. G. B. \& McCumber, D. E. Propagation of a gaussian light pulse through an anomalous dispersion medium. Phys. Rev. A 1, 305-313 (1970).

7. Chu, S. \& Wong, S. Linear pulse propagation in an absorbing medium. Phys. Rev. Lett. 48, 738-741 (1982).

8. Akulshin, A. M., Barreiro, S. \& Lezama, A. Steep anomalous dispersion in coherently prepared Rb vapor. Phys. Rev. Lett. 83, 4277-4280 (1999).

9. Basov, N. G., Ambartsumyan, R. V., Zuev, V. S., Kryukov, P. G. \& Letokhov, V. S. Nonlinear amplification of light pulses. Sov. Phys. JETP 23, 16-22 (1966).

10. Casperson, L. \& Yariv, A. Pulse propagation in a high-gain medium. Phys. Rev. Lett. 26, 293-295 (1971).

11. Icsevgi, A. \& Lamb, W. E. Propagation of light pulses in a laser amplifier. Phys. Rev. 185, 517-545 (1969).

12. Picholle, E., Montes, C., Leycuras, C., Legrand, O. \& Botineau, J. Observation of dissipative superluminous solitons in a Brillouin fiber ring laser. Phys. Rev. Lett. 66, 1454-1457 (1991).

13. Fisher, D. L. \& Tajima, T. Superluminous laser pulse in an active medium. Phys. Rev. Lett. 71, 43384341 (1993).

14. Chiao, R. Y. Superluminal (but causal) propagation of wave packets in transparent media with inverted atomic populations. Phys. Rev. A 48, R34-R37 (1993).

15. Bolda, E. L., Chiao, R. Y. \& Garrison, J. C. Two theorems for the group velocity in dispersive media. Phys. Rev. A 48, 3890-3894 (1993).

16. Steinberg, A. M. \& Chiao, R. Y. Dispersionless, highly superluminal propagation in a medium with a gain doublet. Phys. Rev. A 49, 2071-2075 (1994).

17. Mitchell, M. W. \& Chiao, R. Y. Causality and negative group delays in a simple bandpass amplifier. Am. J. Phys. 66, 14-19 (1998).

18. Bolda, E., Garrison, J. C. \& Chiao, R. Y. Optical pulse propagation at negative group velocities due to a nearby gain line. Phys. Rev. A 49, 2938-2947 (1994).

19. Steinberg, A. M., Kwiat, P. G. \& Chiao, R. Y. Measurement of the single-photon tunneling time. Phys. Rev. Lett. 71, 708-711 (1993).

20. Harris, S. E. Electromagnetically induced transparency. Phys. Today 50, 36-42 (1997).

21. Scully, M. O. \& Zubairy, M. S. Quantum Optics (Cambridge Univ. Press, 1997).

22. Xiao, M., Li, Y.-Q., Jin, S.-Z. \& Gea-Banacloche, J. Measurement of dispersion properties of electromagnetically induced transparency in rubidium atoms. Phys. Rev. Lett. 74, 666-669 (1995).

23. Hau, L. V., Harris, S. E., Dutton, Z. \& Behroozi, C. H. Light speed reduction to 17 meters per second in an untracold atomic gas. Nature 397, 594-598 (1999).

24. Kash, M. M. et al. Ultraslow group velocity and enhanced nonlinear optical effects in a coherently driven hot atomic gas. Phys. Rev. Lett. 82, 5229-5232 (1999).

25. Budker, D., Kimball, D. F., Rochester, S. M. \& Yashchuk, V. V. Nonlinear magneto-optics and reduced group velocity of light in atomic vapor with slow ground state relaxation. Phys. Rev. Lett. 83, 17671770 (1999).

\section{Acknowledgements}

We thank R. A. Linke for several stimulating discussions. We thank D. K. Walter, W. Happer, J. A. Giordmaine, D. J. Chadi, S. A. Solin, R. Y. Chiao, S. E. Harris and E. S. Polzik for helpful discussions. We thank E. B. Alexandrov and N. P. Bigelow for the use of the paraffin-coated caesium cells.

Correspondence and requests for materials should be addressed to L.J.W. (e-mail: Lwan@research.nj.nec.com). 
30. Christofori, G. \& Semb, H. The role of the cell-adhesion molecule E-cadherin as a tumour-suppressor gene. Trends Biochem. Sci. 24, 73-76 (1999).

\section{Acknowledgements}

We thank L. Meyaard for critically reading the manuscript and members of the Clevers C. Kenyon for tips on staining muIs 2 animals for $\beta$-galactosidase expression; and A. Fire for pPD49.78. This work was supported in part by an NIH grant to M.H and PIONEER and Program grants from NWO Medische Wefenschappen to H.C.

Correspondence and requests for materials should be addressed to H.K. (e-mail: R.Korswagen@lab.azu.nl). laboratory for helpful discussions; C. Kenyon for the $m a b-5$ reporter $m u I s 2$; Q. Ch'ng and

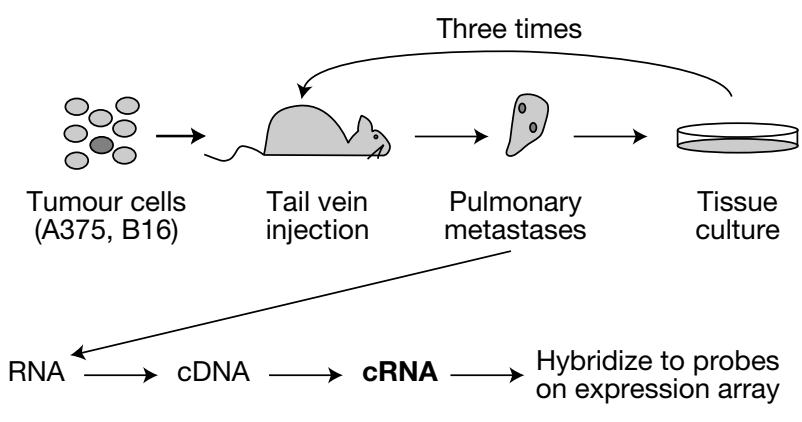

Analysis

Compare parental tumour cell line (A375P or B16F0) grown subcutaneously with the pulmonary metastases (A375M1, M2, SM or B16F1, F2, F3)

Figure 1 In vivo selection scheme. Poorly metastatic melanoma cell lines (human A375P or mouse B16F0) were injected intravenously into the tail veins of host mice and pulmonary metastases were isolated. Either these metastases were minced and grown in tissue culture (to be injected into additional host mice) or RNA was extracted to prepare the labelled cRNA used to hybridize to the oligonucleotide arrays. The procedure to select for highly metastatic tumour cells was repeated two (A375) or three (B16) times. A375SM cells were previously derived in a similar manner ${ }^{11}$.

* Howard Hughes Medical Institute, Centre for Cancer Research, and

\| Department of Biology, Massachusetts Institute of Technology,

77 Massachusetts Avenue, Cambridge, Massachusetts 02139, USA

$\ddagger$ Whitehead Institute/MIT Centre for Genome Research, 9 Cambridge Centre, Cambridge, Massachusetts 02142, USA

$\S$ Dana-Farber Cancer Institute, 44 Binney Street, Boston, Massachusetts 02115, USA

The most damaging change during cancer progression is the switch from a locally growing tumour to a metastatic killer. This switch is believed to involve numerous alterations that allow tumour cells to complete the complex series of events needed for metastasis ${ }^{1}$. Relatively few genes have been implicated in these events ${ }^{2-5}$. Here we use an in vivo selection scheme to select highly metastatic melanoma cells. By analysing these cells on DNA arrays, we define a pattern of gene expression that correlates with progression to a metastatic phenotype. In particular, we show enhanced expression of several genes involved in extracellular matrix assembly and of a second set of genes that regulate, either directly or indirectly, the actin-based cytoskeleton. One of these, the small GTPase RhoC, enhances metastasis when overexpressed, whereas a dominant-negative Rho inhibits metastasis. Analysis of the phenotype of cells expressing dominant-negative Rho or RhoC indicates that RhoC is important in tumour cell invasion. The genomic approach allows us to identify families of genes involved in a process, not just single genes, and can indicate which molecular and cellular events might be important in complex biological processes such as metastasis.

To provide insight into the pattern of gene expression that allows tumours to metastasize, we compared the gene expression profile of melanoma variants with low or high metastatic potential. As shown in Fig. 1, the system involves the in vivo selection of highly metastatic melanoma cells from a population of poorly metastatic tumour cells ${ }^{6}$. When nude mice were injected intravenously with amelanotic human A375P tumour cells, relatively few pulmonary metastases were observed (Fig. 2a). When these rare metastases were dissected free from the lungs and the cells grown in tissue culture, however, the resulting cells showed enhanced metastatic capacity, confirming that highly metastatic cells can be selected from a heterogeneous population of poorly metastatic tumour cells ${ }^{7}$. Furthermore, if successive metastases (designated M1 and M2) were isolated, expanded in tissue culture, and re-introduced into

$\dagger$ Present address: Millennium Predictive Medicine, One Kendall Square, Cambridge, Massachusett 02139, USA. host mice as shown in Fig. 1, significantly more pulmonary metastases were observed (Fig. 2b). When mouse B16F0 melanoma cells were subjected to this same in vivo selection scheme, highly metastatic pulmonary tumours (designated F1, F2 and F3) were isolated, as previously described for this cell line ${ }^{6}$. When the poorly metastatic A375P or B16F0 and the in vivo-selected metastatic A375 or B16 cells were grown as subcutaneous tumours, there was no observable difference in tumour size (see Supplementary Information), indicating that we had selected for a difference in metastatic, but not tumorigenic, properties of the melanomas. These results support the hypothesis that specific gene products can regulate metastasis without altering the growth properties of a tumour ${ }^{8}$. Therefore, we sought to identify metastasis-specific genes using a functional genomics approach.

RNAs extracted from these pulmonary metastases and from the parental $\mathrm{A} 375 \mathrm{P}$ and $\mathrm{B} 16 \mathrm{~F} 0$ lines grown as subcutaneous tumours were used to prepare complementary RNAs (cRNAs), which were hybridized to oligonucleotide microarrays (human: 7,070 genes; mouse: 6,347 genes, with around 50\% overlap in the genes represented) to determine the array of differentially expressed genes (Fig. 1). The entire data set is available at our web site at http://www.genome.wi.mit.edu/MPR and in Supplementary Information. Table 1 lists those genes expressed at consistently higher levels in pulmonary metastases derived from the A375P line (M1, M2 and SM) and the mouse B16F0 line (F1, F2 and F3). To ensure that the enhanced expression of these genes in the pulmonary metastases was not due solely to the influence of the microenvironment in which the metastatic cells were growing, we also grew metastatic A375SM cells subcutaneously and compared their expression profile with that of subcutaneous A375P tumours. We found that 15 of the 16 genes continued to show enhanced expression when metastatic A375 cells were grown as a subcutaneous tumour (see Supplementary Information), indicating that the expression of these genes is intrinsic to the metastatic cells. Note, however, that the tumour microenvironment may help to regulate the absolute level of gene expression.

As the set of genes represented on the human and mouse arrays partially overlapped, some signals appeared in both species (Table 1). Three genes, fibronectin, RhoC and thymosin $\beta 4$, were expressed at higher levels ( $\geq 2.5$-fold) in all three metastases selected from both the human A375 and mouse B16 cell lines. Enhanced expression of these three genes in the pulmonary metastases was 


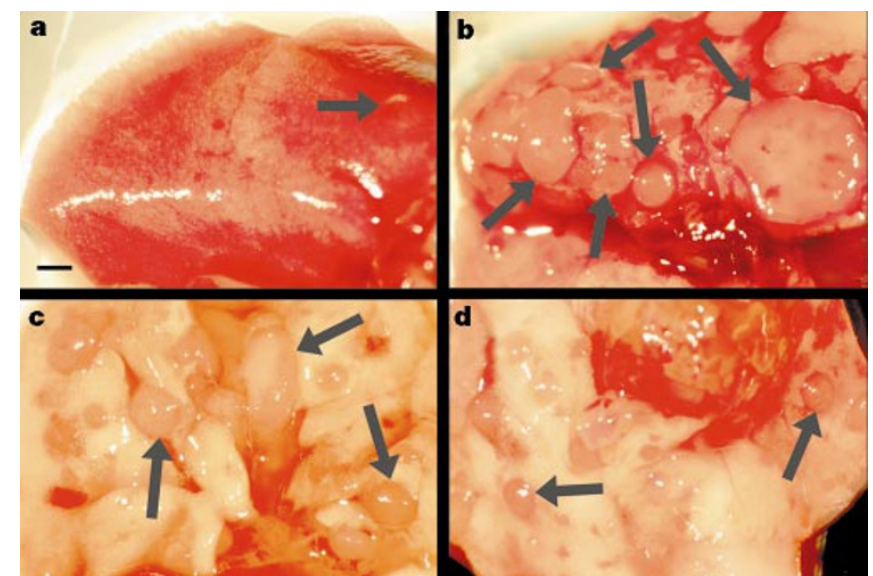

Figure 2 Pulmonary metastases in lungs of mice injected with tumour cell lines. a, A375P cells; b, A375M; c, A375P-RhoC; d, A375M-dnRho. Arrows indicate representative metastatic nodules. Scale bar, $1 \mathrm{~mm}$.

confirmed by RNAse protection (see Supplementary Information). Note that this assay will detect only human RNAs (from the tumour cells) and not mouse RNAs from stromal or vascular cells. This confirms that changes in expression of these three genes occur in the tumour cells rather than in host tissue. This is also probably true for all the oligonucleotide array data given the sequence divergence between species (Table 1).

Fibronectin is an extracellular glycoprotein that serves as a ligand for the integrin family of cell adhesion receptors and regulates cytoskeletal organization. Fibronectin expression has been linked with tumorigenesis ${ }^{9}$ and metastasis ${ }^{10}$, although these studies are only correlative. Peptides that mimic the cell adhesive region of fibronectin are, however, known to inhibit metastasis ${ }^{11}$, which may indicate that tumour cells must interact with molecules such as fibronectin to metastasize. RhoC is a member of the Rho GTPase family that can regulate many cellular functions, most notably cytoskeletal organization, in response to extracellular factors ${ }^{12}$. Enhanced expression of RhoC has been reported to correlate with the progression of pancreatic adenocarcinomas to a metastatic phenotype $^{13}$. Thymosin $\beta 4$ is an actin-sequestering protein that regulates actin polymerization; its expression in renal tumours has been correlated with malignancy ${ }^{14}$. Expression of two other family members, thymosin $\beta 10$ and thymosin $\beta 15$, also correlates with metastasis $^{4,15}$. Other regulators of the cytoskeleton also appear on the list, including $\alpha$-catenin and expressed sequence tags (ESTs) for $\alpha$-actinin 1 and $\alpha$-centractin. The altered expression of so many genes whose products regulate the actin cytoskeleton either directly or indirectly indicates that cytoskeletal organization may be important in tumour metastasis.

Also prominent on the list in Table 1 are several genes that encode extracellular matrix (ECM) proteins, as well as molecules that regulate their assembly. In addition to fibronectin, two collagen subunits, $\alpha 2$ (I) and $\alpha 1$ (III), the matrix Gla protein, fibromodulin and biglycan also are expressed at higher levels in the metastatic melanomas. Previous studies of matrix Gla protein have shown that it is overexpressed in breast carcinoma cell lines relative to normal breast epithelial cells ${ }^{16}$ and collagen expression has been correlated with the invasive potential of ocular melanomas ${ }^{10}$, but expression of the small interstitial proteoglycans biglycan and fibromodulin (which regulate collagen fibril formation ${ }^{17,18}$ ) has yet to be linked to tumour progression. These findings support hypotheses that enhanced expression of ECM proteins may promote tumour cell survival or angiogenesis ${ }^{19}$.

Table 1 Enhanced gene expression in metastatic melanomas

\begin{tabular}{|c|c|c|c|c|c|c|c|c|c|c|c|c|}
\hline \multirow[b]{2}{*}{ Gene name } & \multicolumn{6}{|c|}{ human A375 } & \multicolumn{6}{|c|}{ mouse B16 } \\
\hline & $\begin{array}{c}\text { Human } \\
\text { acc. number }\end{array}$ & Ch. no. & $P$ & M1 & M2 & SM & $\begin{array}{c}\text { Mouse acc. } \\
\text { number }\end{array}$ & FO & $\mathrm{F} 1$ & $\mathrm{~F} 2$ & F3 & Nuc. ident. \\
\hline Fibronectin & X02761 & 2 & 1 & 10.1 & 3.2 & 4.0 & M18194 & A & 2.8 & 2.8 & 2.8 & $93 \%$ \\
\hline RhoC & L25081 & 1 & $A$ & 4.7 & 3.1 & 2.8 & X80638 & A & 2.9 & 4.9 & 2.5 & $91 \%$ \\
\hline Thymosin $\beta 4$ & M17733 & $x$ & 1 & 3.3 & 3.6 & 3.5 & W41883 & 1 & 4.1 & 3.5 & 3.5 & $92 \%$ \\
\hline t-PA & K03021 & 8 & $A$ & 5.2 & 9.6 & 5.2 & J03520 & $A$ & $A$ & $A$ & $A$ & $81 \%$ \\
\hline Angiopoietin1 & D13628 & 8 & 1 & 4.3 & 9.4 & 3.3 & U83509 & & & & * & \\
\hline IEX-1/Glu96 & S81914 & 6 & 1 & 9.1 & 3.3 & 4.5 & X67644 & 1 & 0.4 & 0.6 & 0.5 & $83 \%$ \\
\hline RTP/NDR1 & D87953 & 8 & 1 & 8.6 & 5.4 & 4.7 & U60593 & 1 & A & 0.7 & 1.5 & $86 \%$ \\
\hline Fibromodulin & U05291 & 1 & $A$ & 8.3 & 4.7 & 8.2 & X94998 & 1 & 2.0 & 2.0 & 1.1 & $80 \%$ \\
\hline Hsp70 & M11717 & 1 & 1 & 7.8 & 4.2 & 5.0 & M20567 & 1 & 2.1 & 1.8 & 1.8 & $80 \%$ \\
\hline IL13 Rec., $\alpha 2$ & U70981 & $x$ & 1 & 7.6 & 2.9 & 3.1 & U65747 & & & & * & \\
\hline Sec61 & L25085 & 9 & 1 & 3.8 & 4.7 & 3.3 & & & & & $\dagger$ & \\
\hline snRNP, poly.pep. C & HG1322- & 9 & 1 & 3.8 & 5.3 & 3.2 & & & & & $\dagger$ & \\
\hline Collagen $1 \alpha 2$ & $\mathrm{Z} 74616$ & 7 & A & 2.5 & 3.6 & 3.6 & X58251 & $A$ & 3.1 & 2.3 & 3.7 & $86 \%$ \\
\hline UBE21 & U45328 & 16 & 1 & 3.6 & 3.4 & 3.4 & & & & & $\dagger$ & \\
\hline KIAA0156 & D63879 & 16 & 1 & 3.6 & 3.4 & 3.4 & & & & & $\dagger$ & \\
\hline TGF $\beta$ superfamily & AB000584 & 19 & 1 & 3.4 & 3.4 & 3.0 & & & & & $\dagger$ & \\
\hline Surfactant protein C & J03890 & & & * & & & M38314 & $A$ & 32 & 12 & 16 & \\
\hline Lysozyme M & & & & $\dagger$ & & & M21050 & $A$ & 20 & 10 & 22 & \\
\hline Matrix Gla prot & X53331 & 12 & 1 & 3.2 & 4.4 & 1.1 & D00613 & 1 & 12 & 11 & 5.4 & $81 \%$ \\
\hline Tsa-1 & & & & $\dagger$ & & & U47737 & $A$ & 9.7 & 6.1 & 7.2 & \\
\hline Collagen III 1 & X06700 & 2 & $A$ & $A$ & $A$ & $A$ & X52046 & $A$ & 8.2 & 5.6 & 5.5 & $89 \%$ \\
\hline Biglycan & J04599 & $x$ & A & A & 3.7 & $A$ & L20276 & $A$ & 3.8 & 4.4 & 6.9 & $87 \%$ \\
\hline$\alpha$-catenin & U03100 & 5 & 1.0 & 1.3 & 1.0 & 1.9 & X59990 & 1 & 3.4 & 3.0 & 5.7 & $91 \%$ \\
\hline Valosin-cont. prot. & AC004472 & & & * & & & Z14044 & 1 & 3.0 & 3.9 & 5.9 & \\
\hline ERK-1 & X60188 & 16 & $A$ & A & A & $A$ & Z14249 & 1 & 2.6 & 2.6 & 3.0 & $85 \%$ \\
\hline$\alpha$-actinin 1 & & & & & & & AA068062 & 1 & 3.6 & 3.3 & 7.3 & \\
\hline calmodulin & & & & & & & AA103356 & A & 4.8 & 6.7 & 5.5 & \\
\hline EIF4 $\gamma$ & & & & & & & AA002277 & A & 4.7 & 3.2 & 2.6 & \\
\hline$\alpha$-centractin & & & & & & & W48490 & 1 & 2.9 & 3.8 & 3.6 & \\
\hline IQGAP1 & & & & & & & AA118739 & A & 3.6 & $\begin{array}{l}0.0 \\
3.5\end{array}$ & 3.2 & \\
\hline cathepsin s & & & & & & & W13263 & $A$ & 2.8 & 2.8 & 3.1 & \\
\hline EF2 & & & & & & & W90866 & 1 & 2.6 & 2.5 & 2.9 & \\
\hline
\end{tabular}

Genes whose expression is consistently enhanced $>2.5$-fold in pulmonary metastases (M1, M2, SM, F1, F2 or F3) compared to poorly metastatic cells (P or F0) grown as subcutaneous tumours. The values for $\mathrm{P}$ and $\mathrm{FO}$ are the average of two experiments performed with subcutaneous tumours from two mice injected with A375P or B16F0 cells. Data are presented as fold expression compared with the poorly



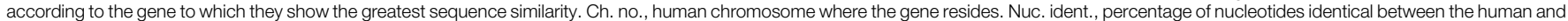
mouse homologues, as determined by BLAST search. The accession number is the GenBank entry from which the oligonucleotide probe sequences were drawn.

* Mouse or human gene homologue exists in the UNIGENE database but was not part of the oligonucleotide probe set.

† No gene homologue was found in the UNIGENE database. 

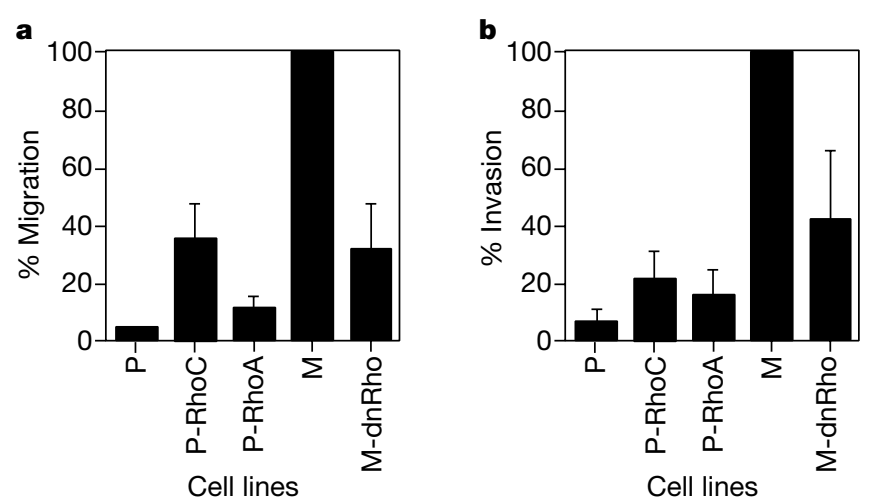

Figure 3 RhoC regulates melanoma cell chemotaxis and invasion. a, Poorly $(\mathrm{P})$ or highly metastatic (M) A375 cells expressing rhoC, rhoA or dominant-negative (dn) rho were induced to migrate towards serum for $16 \mathrm{~h}$. Over $25 \%$ of the plated $\mathrm{A} 375 \mathrm{M}$ cells migrated within this time. Each bar represents the mean \pm s.e.m. of four experiments done in duplicate. $\mathbf{b}$, The cell lines described above were induced to invade matrigel-coated membranes for $48 \mathrm{~h}$. Over $30 \%$ of the plated A375M cells invaded within this time. Each bar represents the mean \pm s.e.m. of three experiments done in duplicate.

Several genes identified in other studies are conspicuous by their absence from our list. Metastasis suppressor genes, such as nm23, KiSS1 and CD82, can inhibit tumour metastasis ${ }^{2}$. In our study all three of these genes were absent in both the parental A375 tumours and in the metastases (see Supplementary Information), indicating that, although expression of these genes may inhibit metastasis, lack of their expression is not sufficient for metastasis. Other genes not found in Table 1 but whose expression correlated with melanoma metastasis in previous studies include the Met tyrosine kinase receptor, matrix metalloproteinases (MMPs) such as MMP2, and the $\beta 3$-integrin subunit ${ }^{20-22}$. In the B16 tumours, Met expression was higher in two of the three metastases but its expression was not detected in any of the A375 tumours, indicating that its expression may not be essential for these tumours to metastasize. Expression of MMP2 and of the $\beta 3$-integrin subunit was not significantly higher in any of the three metastases (see Supplementary Information), but their expression in both the parental and metastatic tumours may be sufficient to allow the tumour cells to metastasize.

Having uncovered 32 genes and ESTs whose expression patterns correlate with metastasis, we wished to investigate the function of one of these genes in this process. Because of its elevated expression in metastases derived from both tumour cell lines, RhoC was chosen to test the hypothesis that these expression studies identify genes essential for metastasis. The full-length human RhoC gene was cloned, subcloned into a retroviral vector and introduced into a retroviral packaging cell line. We used retroviral particles to infect the poorly metastatic A375P cells, and selected cells expressing high levels of RhoC by fluorescence-activated cell sorting (FACS). These cells, designated A375P-RhoC (expressing RhoC at 20 times the level expressed in A375M cells), were subjected to the experimental metastasis assay. As seen in Fig. 2c and Table 2, overexpression of RhoC markedly enhanced metastasis in this system.

Next we tested whether we could inhibit metastasis by expressing a known dominant-inhibitory Rho mutant (N19Rho) ${ }^{23}$ in the highly metastatic A375M cells (a pool of M1, M2 and SM cells). This mutant is analogous to the N17Ras mutant that blocks Ras signalling ${ }^{24}$. Ras dominant-negatives are actually antagonists of the guanine-nucleotide exchange factors (GNEFs) for Ras, rather than of Ras itself ${ }^{23}$ and it is believed that dominant-negative RhoA antagonizes Rho GNEFs, thereby inhibiting RhoC. Expression of N19RhoA in the A375M cells markedly suppressed the generation of metastases when these cells were subjected to the experimental metastasis assay (Fig. 2d and Table 2), indicating that Rho activity may be necessary, and RhoC sufficient, for metastasis in these melanoma lines.


Figure 4 Metastatic capacity of A375 melanoma cells correlates with cell morphology. Poorly (a) or highly metastatic (c) A375 cells expressing rhoC (b) or dominant-negative rho (d) were plated on glass coverslips for $16 \mathrm{~h}$ at $37^{\circ} \mathrm{C}$, then fixed and stained with phalloidin to detect F-actin.

Having established a causal role for RhoC in metastasis, we set out to investigate how RhoC might regulate the ability of tumour cells to metastasize. Tumour cells must complete a complex series of steps to metastasize, one of the most basic of which is cell growth. Rho GTPases affect several aspects of growth control ${ }^{12}$, so it was possible that RhoC might control tumour metastasis by regulating cell proliferation. We assayed the A375P, A375P-RhoC, A375M and A375M-dnRho cells for in vitro proliferation and in vivo tumorigenesis (see Supplementary Information). Proliferation in either assay was not significantly changed by altering RhoC expression or Rho activity, indicating that RhoC may regulate metastasis by a mechanism other than controlling cell proliferation.

Another function of Rho-family GTPases is to control cytoskeletal organization in response to extracellular factors ${ }^{12}$. Cytoskeletal proteins are known effectors for events essential for cell motility ${ }^{25}$. Therefore, RhoC may control metastasis by regulating cell motility. Metastatic A375M cells were more migratory (Fig. 3a) and more invasive (Fig. 3b) than the poorly metastatic A375P cells. Furthermore, RhoC could enhance the migratory and invasive capacity of the A375P cells, whereas dnRho inhibited motility and invasion of the $\mathrm{A} 375 \mathrm{M}$ cells, indicating that RhoC may regulate metastasis by controlling cytoskeletal events essential for motility. We also observed that RhoC could induce in A375P cells an elongated morphology similar to that of A375M cells, while dnRho expression suppressed this morphology (Fig. 4). Another morphological difference noted in the A375M cells, the seruminduced formation of filopodia, also correlated with the metastatic capacity of these cells. However, filopodial protrusions (which are regulated by the Rho subfamily member Cdc42 (ref. 12) were not altered by expression of RhoC or dnRho (data not shown), indicating that regulation of these actin-based structures may occur upstream (or independently) of Rho.

We have identified RhoC as essential for tumour metastasis. Compared with RhoA, the canonical family member, little is

\section{Table 2 Pulmonary metastases}

Cell line

A375P

A375P-RhoC

A375M

A375M-dnRho

No. of metastases

No. of mice

Numbers of pulmonary metastases identified on the surface of the lungs of mice injected with A375P, A375P-RhoC, A375M or A375M-dnRho cells. 
known about RhoC. RhoA and RhoC are highly homologous, with only six non-conservative amino-acid substitutions, all in the carboxy-terminal end of the molecules. It might be thought that RhoA should be able to enhance tumour metastasis. However, RhoA is expressed at equivalent levels in both the poorly and highly metastatic tumours (see Supplementary Information), indicating that the level of RhoA expression in the A375 cells is not sufficient for metastasis. Furthermore, when expressed at equivalent levels, RhoC was a better motogen than was RhoA (Fig. 3). These results indicate that there may be a functional difference between Rho subfamily members that requires further investigation. Finally, the observation that expression of a single gene is sufficient to induce metastasis is perhaps surprising, given that metastasis is such a complex process. We suspect, however, that many cells within the heterogeneous tumorigenic A375P population may be genetically primed for metastasis so that introduction of a single gene (such as RhoC) which affects a process essential for metastasis is sufficient for metastasis. We are currently examining whether RhoC is capable of inducing metastasis in other tumorigenic cells.

\section{Methods}

\section{Cell lines}

The A375 (ATCC\#CRL-1619) and B16 (ATCC\#CRL-6322) cell lines were maintained as described $^{7}$. Cells were harvested by trypsinization, washed in PBS and diluted to $2.5 \times 10^{6}$ cells per ml for A375 cells and $2.5 \times 10^{5}$ cells per $\mathrm{ml}$ for B16 cells.

\section{Experimental metastasis assay}

A375 cells were injected either intravenously $(0.2 \mathrm{ml})$ into the lateral tail vein or subcutaneously $(0.1 \mathrm{ml})$ into the dorsal flank of nude mice, and B16 cells were injected into syngeneic C57BL/6 mice. Three (for B16) to eight (for A375) weeks after injection the mice were killed; the lungs were removed and washed and the pulmonary metastases on the lung surface were counted under a dissecting microscope. Metastatic nodules were removed aseptically, minced and grown in vitro, or snap-frozen in liquid nitrogen to purify RNA.

\section{Tumours and tumour-derived cell lines}

A375M1, M2 and SM lines were selected using the experimental metastasis assay for their enhanced ability to form experimental pulmonary metastases ${ }^{6}$. Line M1 was derived from metastases isolated from mice injected intravenously with the A375P cells, line M2 from mice injected with A375M1 cells, and line SM was a gift from I. Fidler and was derived by an identical selection procedure ${ }^{7}$. B16 lines were derived in an identical manner, with F1 cells derived from B16F0 cells, F2 from B16F1 cells and F3 from B16F2 cells. The A375M cell line is a pool of cells from A375M1, M2 and SM cells. A375P and A375M cells used in retroviral gene transfer studies were transfected with a plasmid containing the ecotropic receptor (a gift from H. Lodish) and selected for neomycin-resistance. Mock-infected or uninfected cells were used as negative controls in the metastasis assays.

\section{Array hybridization}

Total RNA was prepared with a Qiagen RNeasy mini-kit according to the manufacturer's instructions. We prepared cRNA for hybridization essentially as described ${ }^{26}$. Oligonucleotide arrays (GeneChip, Affymetrix) composed of 7,070 human (HUM 6.8K) or 6,347 mouse (MUR 6K) genes and ESTs were used for hybridization according to the manufacturer's instructions. Arrays were scanned using an Affymetrix confocal scanner and analysed using GeneChip 3.0 software (Affymetrix). Intensity values were scaled so that the overall fluorescence intensity of each chip of the same type was equivalent. For a gene to be selected as induced, it has to be expressed in all three metastatic samples at least 2.5 times higher than in the poorly metastatic sample, with experiments done in duplicate. Where expression in the poorly metastatic sample was below baseline (set at 20, the point below which changes in expression could be determined with high confidence), it was determined to be absent and was set to 20 .

\section{Retroviral gene transfer}

An EcoRl fragment of pCR-BluntII-RhoC containing the entire coding region of human RhoC (see Supplementary Information) was inserted into the EcoR1 site of the retroviral bicistronic expression vector pMX-IRES-GFP (pMIG; ref. 27) containing enhanced green fluorescent protein (GFP) as an expression marker. An EcoR1 fragment of pEXV-RhoA or pEXV-N19RhoA (a dominant-negative Rho mutant, dnRho) was inserted into the EcoR1 site of pMIG. We transfected pMIG-RhoC, pMIG-RhoA and pMIG-dnRho into 293T cellderived retroviral producer lines (Phoenix cells) as described (see www.stanford.edu/ group/nolan/) and A375P or M cells were infected. Cells were sorted by FACStar (BectonDickinson) according to their GFP levels. RNAse protection assays showed that RhoC was expressed at 5- to 50-fold higher levels than in the A375M cells, whereas RhoC and RhoA were expressed at similar levels in the A375P-RhoC and A375P-RhoA cells (as determined by GFP expression).

\section{Chemotaxis and invasion assays}

Cell migration and invasion assays were performed using $8.0-\mu \mathrm{m}$ pore size Transwell inserts (Costar Corporation) or Biocoat Matrigel invasion chambers (Becton-Dickinson), respectively ${ }^{28}$. Each data point represents the average of 3 or 4 individual experiments, done in duplicate, and error bars represent the standard error of the mean.

\section{Immunofluorescence}

Adherent cells were fixed, permeabilized and stained as described ${ }^{29}$.

\section{Received 31 January; accepted 15 May 2000}

1. Van Noorden, C. J. F., Meade-Tollin, L. C. \& Bosman, F. T. Metastasis. Am. Sci. 86, 130-141 (1998).

2. Fidler, I. J. \& Radinsky, R. Search for genes that suppress cancer metastasis. J. Natl Cancer Inst. 88, 1700-1703 (1996)

3. Roberts, D. D. Regulation of tumor growth and metastasis by thrombospondin-1. FASEB J. 10, 11831191 (1996).

4. Bao, L. et al. Thymosin $\beta 15$ : a novel regulator of tumor cell motility upregulated in metastatic prostate cancer. Nature Med. 2, 1322-1328 (1996).

5. Habets, G. G. M. et al. Identification of an invasion-inducing gene, Tiam-1, that encodes a protein with homology to GDP-GTP exchangers for rho-like proteins. Cell 77, 537-549 (1994).

6. Fidler, I. J. Selection of successive tumour lines for metastasis. Nature 242, 148-149 (1973).

7. Kozlowski, J. M., Hart, I. R., Fidler, I. J. \& Hanna, N. A human melanoma cell line heterogeneous with respect to metastatic capacity in athymic nude mice. J. Natl Cancer Inst. 72, 913-917 (1984).

8. Welch, D. R. et al. Microcell-mediated transfer of chromosome 6 into metastatic human C8161 melanoma cells suppresses metastasis but does not inhibit tumorigenicity. Oncogene 9, 255-262 (1994).

9. Zhang, L. et al. Gene expression profiles in normal and cancer cells. Science 276, 1268-1272 (1997).

10. Maniotis, A. J. et al. Vascular channel formation by human melanoma cells in vivo and in vitro: vasculogenic mimicry. Am. J. Pathol. 155, 739-752 (1999).

11. Humphries, M. J., Olden, K. \& Yamada, K. M. A synthetic peptide from fibronectin inhibits experimental metastasis of murine melanoma cells. Science 233, 467-469 (1986).

12. Van Aelst, L. \& D'Souza-Schorey, C. Rho GTPases and signaling networks. Genes Dev. 11, 2295-2322 (1997).

13. Suwa, H. et al. Overexpression of the rhoC gene correlates with progression of ductal adenocarcinoma of the pancreas. Br. J. Cancer 77, 147-152 (1998).

14. Hall, A. K. Differential expression of thymosin genes in human tumors and in the developing human kidney. Int. J. Cancer 48, 672-677 (1991).

15. Weterman, M. A. J., van Muijen, G. N. P., Ruiter, D. J. \& Bloemers, H. P. J. Thymosin $\beta-10$ expression in melanoma cell lines and melanocytic lesions: a new progression marker for human cutaneous melanoma. Int. J. Cancer 53, 278-284 (1993).

16. Chen, L., O'Bryan, J. P., Smith, H. S. \& Liu, E. Overexpression of matrix Gla protein mRNA in malignant human breast cells: isolation by differential cDNA hybridization. Oncogene 5, 1391-1395 (1990).

17. Schonherr, E. et al. Interaction of biglycan with type 1 collagen. J. Biol. Chem. 270, 2776-2783 (1995).

18. Svensson, L. et al. Fibromodulin-null mice have abnormal collagen fibrils, tissue organization, and altered lumican deposition in tendon. J. Biol. Chem. 274, 9636-9647 (1999).

19. Ruoslahti, E. Fibronectin and its integrin receptors in cancer. Adv. Cancer Res. 76, 1-20 (1999).

20. Jeffers, M., Rong, S. \& Vande Woude, G. F. Enhanced tumorigenicity and invasion-metastasis by hepatocyte growth factor/scatter factor-Met signalling human cells concomitant with induction of the urokinase proteolysis network. Mol. Cell. Biol. 16, 1115-1125 (1996).

21. Chambers, A. F. \& Matrisian, L. M. Changing views of the role of matrix metalloproteinases in metastasis. J. Natl Cancer Inst. 89, 1260-1270 (1997)

22. Albelda, S. M. et al. Integrin distribution in malignant melanoma: association of the $\beta 3$ subunit with tumor progression. Cancer Res. 50, 6757-6764 (1990).

23. Quilliam, L. A., Khosravi-Far, R., Huff, S. Y. \& Der, C. J. Guanine nucleotide exchange factors: activators of the Ras superfamily of proteins. BioEssays 17, 395-404 (1995).

24. Feig, L. A. \& Cooper, G. M. Inhibition of NIH $3 \mathrm{~T} 3$ cell proliferation by a mutant ras protein with preferential affinity for GDP. Mol. Cell. Biol. 8, 3235-3243 (1988).

25. Lauffenburger, D. A. \& Horwitz, A. F. Cell migration: a physically integrated molecular process. Cell 84, 359-369 (1996).

26. Fambrough, D., McClure, K., Kazlauskas, A. \& Lander, E. S. Diverse signaling pathways activated by growth factor receptors induce broadly overlapping, rather than independent, sets of genes. Cell 97, 727-741 (1999).

27. Liu, X. et al. Transforming growth factor $\beta$-induced phosphorylation of Smad 3 is required for growth inhibition and transcriptional induction in epithelial cells. Proc. Natl Acad. Sci. USA 94, 10669-10674 (1997)

28. Yebra, M. et al. Requirement of receptor-bound urokinase-type plasminogen activator for integrin avB5-directed cell migration. J. Biol. Chem. 271, 29393-29399 (1996).

29. Clark, E. A., King, W. G., Brugge, J. S., Symons, M. \& Hynes, R. O. Integrin-mediated signals regulated by members of the rho family of GTPases. J. Cell Biol. 142, 573-586 (1998).

Supplementary information is available on Nature's World-Wide Web site (http://www.nature.com) or as paper copy from the London editorial office of Nature.

\section{Acknowledgements}

We thank C. Huard, C. Whittaker, S. Robinson, J. Lively, D. Hirsch, D. Crowley and P. Tamayo for technical assistance and advice, and H. Lodish, G. Nolan and J. Fidler, for reagents. This work was supported in part by grants from the National Cancer Institute (to R.O.H), Affymetrix, Inc., Bristol-Myers Squibb and Millenium Pharmaceuticals (to E.S.L.), and a Merck/MIT postdoctoral fellowship (to E.A.C.). R.O.H. is an investigator and E.A.C. was an associate of the Howard Hughes Medical Institute.

Correspondence and requests for materials should be addressed to R.O.H (e-mail: rohynes@mit.edu). 


\section{Endogenous cannabinoids mediate retrograde signalling at hippocampal synapses}

\section{Rachel I. Wilson \& Roger A. Nicoll}

Departments of Cellular and Molecular Pharmacology and Physiology, University of California, San Francisco, California 94143, USA

Marijuana affects brain function primarily by activating the Gprotein-coupled cannabinoid receptor-1 $(\mathrm{CB} 1)^{1-3}$, which is expressed throughout the brain at high levels ${ }^{4}$. Two endogenous lipids, anandamide and 2-arachidonylglycerol (2-AG), have been identified as CB1 ligands $s^{5,6}$. Depolarized hippocampal neurons rapidly release both anandamide and $2-\mathrm{AG}$ in a $\mathrm{Ca}^{2+}$-dependent manner ${ }^{6-8}$. In the hippocampus, $\mathrm{CB} 1$ is expressed mainly by GABA ( $\gamma$-aminobutyric acid)-mediated inhibitory interneurons, where CB1 clusters on the axon terminal ${ }^{9-11}$. A synthetic CB1 agonist depresses GABA release from hippocampal slices ${ }^{10,12}$. These findings indicate that the function of endogenous cannabinoids released by depolarized hippocampal neurons might be to downregulate GABA release. Here we show that the transient suppression of GABA-mediated transmission that follows depolarization of hippocampal pyramidal neurons ${ }^{13}$ is mediated by retrograde signalling through release of endogenous cannabinoids. Signalling by the endocannabinoid system thus represents a mechanism by which neurons can communicate backwards across synapses to modulate their inputs.

Three properties of 'depolarization-induced suppression of inhibition' (DSI) ${ }^{13}$ suggested a role for cannabinoids in DSI. First, DSI, like endocannabinoid synthesis, requires $\mathrm{Ca}^{2+}$ influx into the postsynaptic neuron ${ }^{14}$. Second, DSI expression is probably presynaptic, as DSI does not affect the sensitivity of the postsynaptic membrane to iontophoresed GABA, or the quantal size of miniature GABAmediated events ${ }^{13,15,16}$, consistent with the localization of CB1 to GABA-containing axon terminals. Last, DSI is blocked by pertussis $\operatorname{toxin}^{17}$, implying a function for $\mathrm{G}_{\mathrm{i}^{-}}$or $\mathrm{G}_{\mathrm{o}}$-coupled receptor such as $\mathrm{CB} 1$. We therefore asked whether $\mathrm{CB} 1$ antagonists block DSI. We recorded from single CA1 pyramidal neurons in hippocampal slices in the whole-cell configuration. Figure 1a shows the transient depression of evoked inhibitory postsynaptic currents (eIPSCs) caused by a brief depolarizing step in the holding potential of a CA1 pyramidal neuron; incubating slices in the CB1 antagonist AM251 (Tocris) abolishes this effect. Figure 1b summarizes the effects of AM251 and another CB1 antagonist, SR141716 (National Institute on Drug Abuse), on DSI magnitude. SR141716 also blocked DSI induced by a train of action potentials $(1 \mathrm{~s}, 20 \mathrm{~Hz})$ rather than a voltage step (data not shown). Acute applications of SR141716 blocked the ability of a depolarizing step to depress eIPSC amplitude without affecting baseline eIPSCs (Fig. 1d). Furthermore, the effect of DSI on inhibitory transmission was mimicked by a synthetic CB1 agonist, WIN55212-2 (RBI), which acutely depressed baseline eIPSC amplitude (Fig. 1e). Pre-incubation in AM251 blocked this depression (eIPSC amplitude $95 \pm 3 \%$ of baseline after $20 \mathrm{~min}$ of WIN55212-2 exposure $(n=6)$; data not shown, $P<0.05$, compared with slices without AM251, paired $t$-test). WIN55212-2 did not affect the DSI-resistant component of the eIPSC (Fig. 1e), and thus occluded DSI.

The natural CB1 ligand 2-AG (Biomol) had only a small effect compared with the synthetic agonist (Fig. 2a), suggesting that 2-AG was not reaching sufficient concentrations in the slice. This is consistent with a recent report that both $2-\mathrm{AG}$ and anandamide are substrates for an endogenous transporter that removes these ligands from the extracellular space ${ }^{18,19}$. Application of AM404
(Tocris), an inhibitor of the anandamide/2-AG transporter, depressed baseline eIPSC amplitude without affecting the DSIresistant component of the eIPSC (Fig. 2b). Pre-incubation of slices in SR141716 abolished the effects of AM404 (Fig. 2c). As AM404 is not itself a CB1 agonist ${ }^{18}$, these results imply that the endogenous substrate for the AM404-sensitive transporter is a cannabinoid that mimics and occludes DSI when allowed to accumulate in the slice. As a CB1 antagonist has no effect on baseline eIPSCs (Fig. 1d), tonic synthesis of endogenous cannabinoids must be normally balanced by uptake, which keeps extracellular cannabinoid levels below that required for $\mathrm{CB} 1$ activation. The kinetics of DSI were not changed as AM404 washed into the slice (Fig. 2d), suggesting that the AM404-sensitive transporter does not clear the retrograde signal rapidly enough to affect DSI decay. The kinetics of DSI probably reflect signal-transduction events inside the presynaptic terminal, or else passive diffusion of cannabinoids away from the site of release.

If an endogenous cannabinoid mediates DSI, then a CB1 agonist and DSI must depress GABA-mediated transmission by the same mechanism. We therefore asked whether DSI and WIN55212-2 act at a similar locus. In those experiments where WIN55212-2 depressed eIPSCs by at least $40 \%$, the amplitude ratio of two closely a
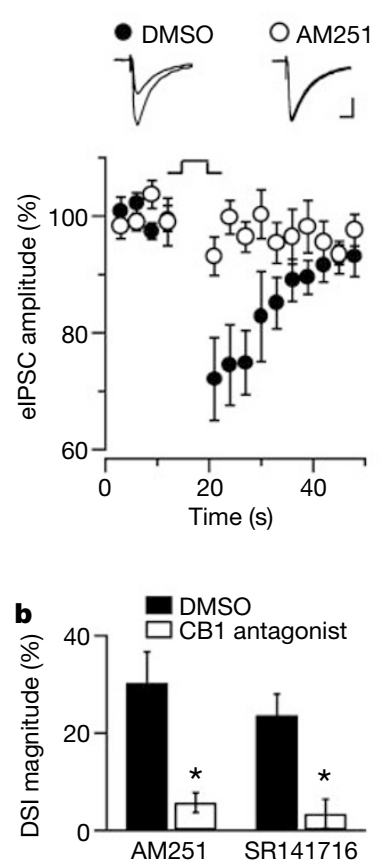
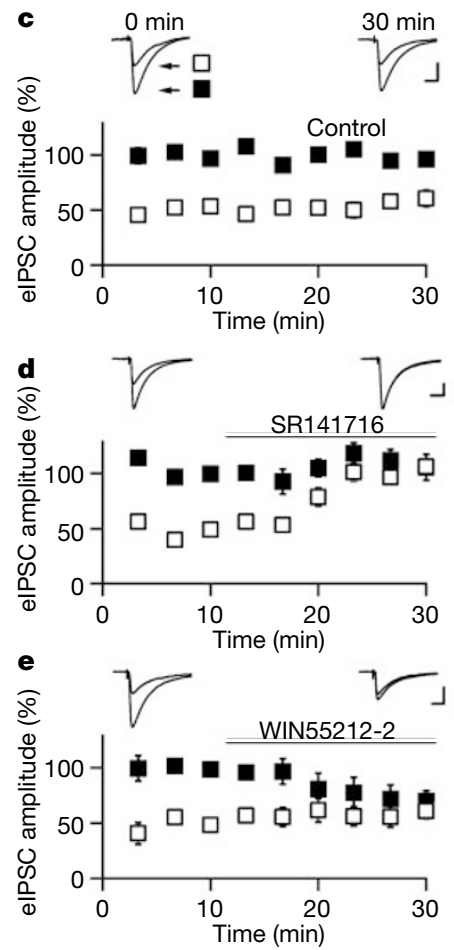

Figure 1 DSI requires endogenous cannabinoids. a, In pyramidal neurons $(n=14)$ from slices pre-incubated in AM251 (2 $\mu \mathrm{M})$, a 5-s depolarizing step results in little or no suppression of elPSCs. Interleaved DMSO controls $(n=17)$ show a robust suppression. Average of 5 trials per cell. Insets show average IPSCs for the $10 \mathrm{~s}$ before and $10 \mathrm{~s}$ after the depolarizing step. $\mathbf{b}$, Effect of AM251 ( $6 \pm 2 \%$ versus $30 \pm 6 \%$ average DSI in AM251 and DMSO alone, respectively) and SR141716 on DSI ( $2 \mu \mathrm{M}: 3 \pm 3 \%$ versus 24 $\pm 4 \%$ average DSI in SR141716 $(n=11)$ and DMSO $(n=10)$ alone, respectively). Asterisk, $P<0.005$. c-e, Control experiments $(n=7)$ (c) show stable DSI over 30 min, whereas SR141716 $(n=6)(\mathbf{d})$ blocks DSI by antagonism, and WIN55212-2 (800 nM: $n=5$ ) (e) blocks DSI by occlusion (average DSI magnitude at $30 \mathrm{~min}$ in controls is $70 \pm 10 \%$ of magnitude during baseline, compared with $11 \pm 10 \%$ in SR141716 slices, and $19 \pm 9 \%$ in WIN55212-2 slices; $P<0.005$ for both treatments). Scale bars: a, c-e, $200 \mathrm{pA}, 20 \mathrm{~ms}$. The statistical test used was the paired $t$-test. Filled squares, elPSC amplitude just before 5-s depolarization; open squares, eIPSC amplitude just after 5-s depolarization. 
spaced eIPSCs (the paired-pulse ratio, PPR) was significantly increased (Fig. 3a). Similarly, DSI also reversibly increased PPR (Fig. 3b), which generally correlates with a local decrease in the probability of vesicular release from the axon terminal. In agreement with a previous study ${ }^{12}$, we also found that WIN55212-2 decreases the frequency of $\mathrm{Ca}^{2+}$-dependent miniature IPSCs (mIPSCs) recorded after blockade of action potentials by tetrodotoxin (TTX), and in the presence of high external potassium and $\mathrm{Ca}^{2+}$ concentrations (Fig. $3 \mathrm{c}, \mathrm{d}$ ). In the same conditions, but without TTX, depolarizing the postsynaptic cell induces a significant decrease in action-potential-driven spontaneous IPSCs (sIPSCs) (Fig. 3e). After adding TTX to the bath, depolarization elicits a similar depression of mIPSC frequency (Fig. 3e, f). Consistent with a presynaptic locus, DSI does not affect mIPSC amplitude ( $100 \pm 3 \%$ of baseline $(n=5)$; data not shown). Thus DSI, like the CB1 agonist, seems to act locally at the presynaptic terminal.

The conclusion that DSI is mediated by endogenous cannabinoids leads to three predictions about the properties of DSI. First, as both anandamide and 2-AG exit the cell by diffusion and/or passive transport, release of the retrograde signal in DSI should not require vesicular fusion ${ }^{8}$. We filled the postsynaptic cell by means of the recording electrode with botulinum toxin E light chain (BTE) while
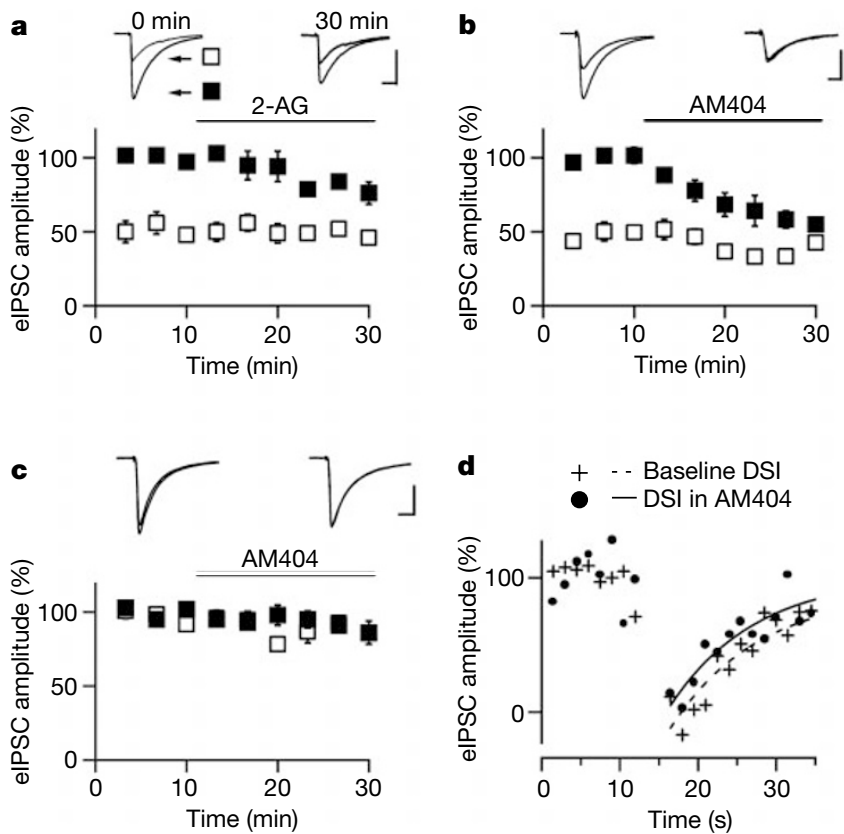

Figure 2 DSI is mimicked and occluded by blocking uptake of endogenous CB1 ligands. a, Effect of 2-AG on elPSCs ( $30 \mu \mathrm{M}$ : $76 \pm 7 \%$ of baseline at $30 \mathrm{~min},(n=6)$ $P<0.05$, compared with controls in Fig. 1C). b, AM404 significantly depresses elPSCs (20 $\mu \mathrm{M}$ : $55 \pm 5 \%$ of baseline at $30 \mathrm{~min},(n=6) P<0.001$, compared with controls in Fig. 1c) and partially occludes DSI (average DSI magnitude at 30 min is $39 \pm 11 \%$ of magnitude during baseline, $P<0.05$, compared with DSI from controls in Fig. 1c). c, Depression of elPSCs by AM404 is antagonized by pre-incubation with SR141716 (elPSC amplitude $85 \pm 4 \%$ of baseline at $30 \mathrm{~min},(n=5) P<0.005$, compared to slices without SR141716). d, AM404 does not significantly affect the kinetics of DSI. Average time course of DSI during baseline period compared to the first 12 min of AM404 wash-in, during which DSI is being progressively occluded, is shown $(n=6$, average of $4-5$ baseline trials and 6 AM404 trials per cell). Lines are single exponential fits to the data (dashed line: baseline DSI, $\tau=22.5 \mathrm{~s}$; solid line: DSI during first $12 \mathrm{~min}$ of AM404 wash-in, $\tau=21.8 \mathrm{~s}$; not significantly different by a within-cell comparison of $\tau, P>0.7$, paired $t$-test). Scale bars: a-c: $200 \mathrm{pA}, 20 \mathrm{~ms}$. $\mathrm{DSI}=\mathrm{DSI}_{\max } \mathrm{e}^{-t / \tau}$ monitoring DSI. BTE proteolytically cleaves both the synaptosomeassociated proteins SNAP-25 and SNAP-23 (refs 20, 21); either of these is a necessary component of the minimal machinery required for all cellular membrane fusion ${ }^{22}$. We found that BTE does not affect the stability of DSI (Fig. 4a). As a control, we incubated recombinant SNAP-25 with BTE in our electrodefilling solution; the concentration of BTE used in our recordings was in ten-fold excess over that required to cleave all the SNAP25 within $1 \mathrm{~h}$ (Fig. 4b). DSI is also unaffected by botulinum toxin B light chain (BTB) (Fig. 4a), which cleaves the vesicle-associated membrane protein VAMP2 and disrupts vesicular fusion in pyramidal cell dendrites when added to the solution in the recording electrode $\mathrm{e}^{23,24}$.

As the trigger for cannabinoid synthesis is cytoplasmic $\mathrm{Ca}^{2+}$, a second prediction is that $\mathrm{Ca}^{2+}$ alone should be sufficient to trigger
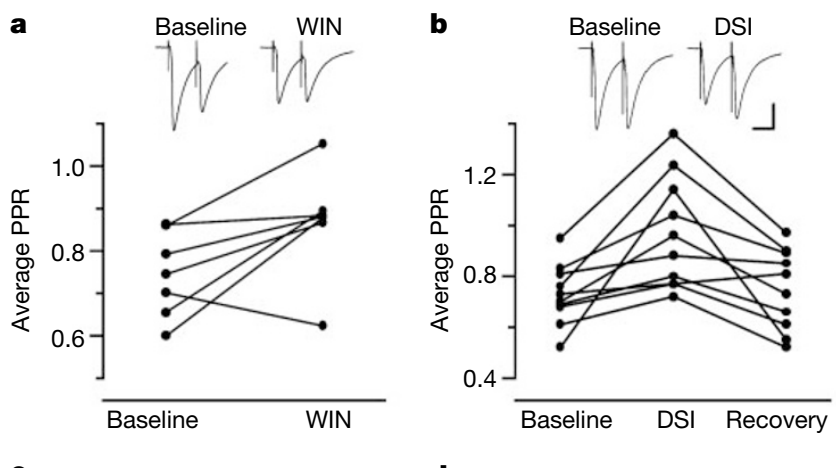

c



d

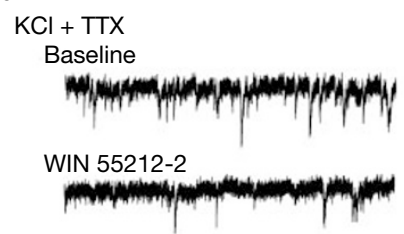

e



$\mathbf{f}$

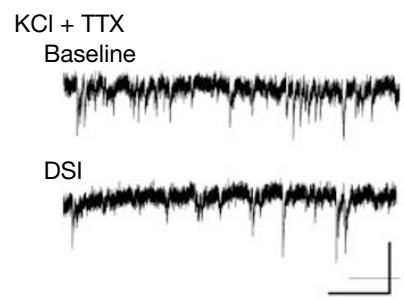

Figure 3 DSI and a CB1 agonist suppress IPSCs by the same mechanism. a, In experiments where WIN55212-2 depressed eIPSCs by at least $40 \%$, the paired-pulse ratio (PPR) was significantly increased (inter-pulse interval $55 \mathrm{~ms} ;(n=7) P<0.05)$. b, DSI reversibly increases the PPR of eIPSCs (inter-pulse interval $55 \mathrm{~ms}$; $(n=10)$ $P<0.01)$. Scale bar: 200 pA, 40 ms. c, d, WIN55212-2 decreases the frequency of mIPSCs recorded in TTX, high external calcium, and high external potassium $(62 \pm 6 \%$ of baseline frequency 20 min after wash-on; $n=6$ ). $\mathrm{CdCl}_{2}$ (Cd) further decreases mIPSC frequency under these conditions ( $40 \pm 12 \%$ of baseline frequency compared with either baseline or WIN55212-2; $n=5$ ) Asterisk, $P<0.005$; double asterisk, $P<0.05$. No change in mIPSC frequency occurred over 20 min when no drug was applied (Sham) (104 $\pm 13 \%$ of baseline; $n=4$ ). $\mathbf{e}, \mathbf{f}$, Under the same conditions of elevated calcium and potassium, but without TTX, depolarizing a pyramidal cell depresses action-potentialdependent sIPSCs (sIPSC frequency $74 \pm 7 \%$ of baseline, $P<0.001$; sIPSC amplitude $87 \pm 6 \%$ of baseline, $(n=5) P<0.05$; data not shown). After adding $T \mathrm{TX}$, depolarizing the same cell causes a similar decrease in mIPSC frequency $(77 \pm 4 \%$ of baseline; $(n=5) P<0.005)$. Scale bar: $50 \mathrm{pA}, 1 \mathrm{~s}$. The statistical test used was the paired $t$-test. 
DSI. We find that the effect of a depolarizing step (Fig. 4c) is indistinguishable from the effect of uncaging $\mathrm{Ca}^{2+}$ from a photolabile chelator when the pyramidal neuron is not depolarized (Fig. 4d). Like $\mathrm{DSI}^{13}, \mathrm{Ca}^{2+}$ uncaging transiently depresses both the frequency and amplitude of sIPSCs (Fig. 4e). $\mathrm{Ca}^{2+}$ uncaging also depresses eIPSCs, an effect that is antagonized by AM251 (Fig. 4f). Like DSI, $\mathrm{Ca}^{2+}$-induced depression is expressed presynaptically, as the amplitude of mIPSCs elicited by sucrose $(150 \mathrm{mM})$ in the presence of TTX $(1 \mu \mathrm{M})$ is unaffected $(97 \pm 4 \%$ of baseline, measured $6 \mathrm{~s}$ after flash $(n=8)$; data not shown).

On the basis of reports that DSI is attenuated by the metabotropic glutamate receptor (mGluR) antagonist (S)- $\alpha$-methyl-4-carboxyphenylglycine $((\mathrm{S})-\mathrm{MCPG})^{25}$, it has been suggested that the retro-
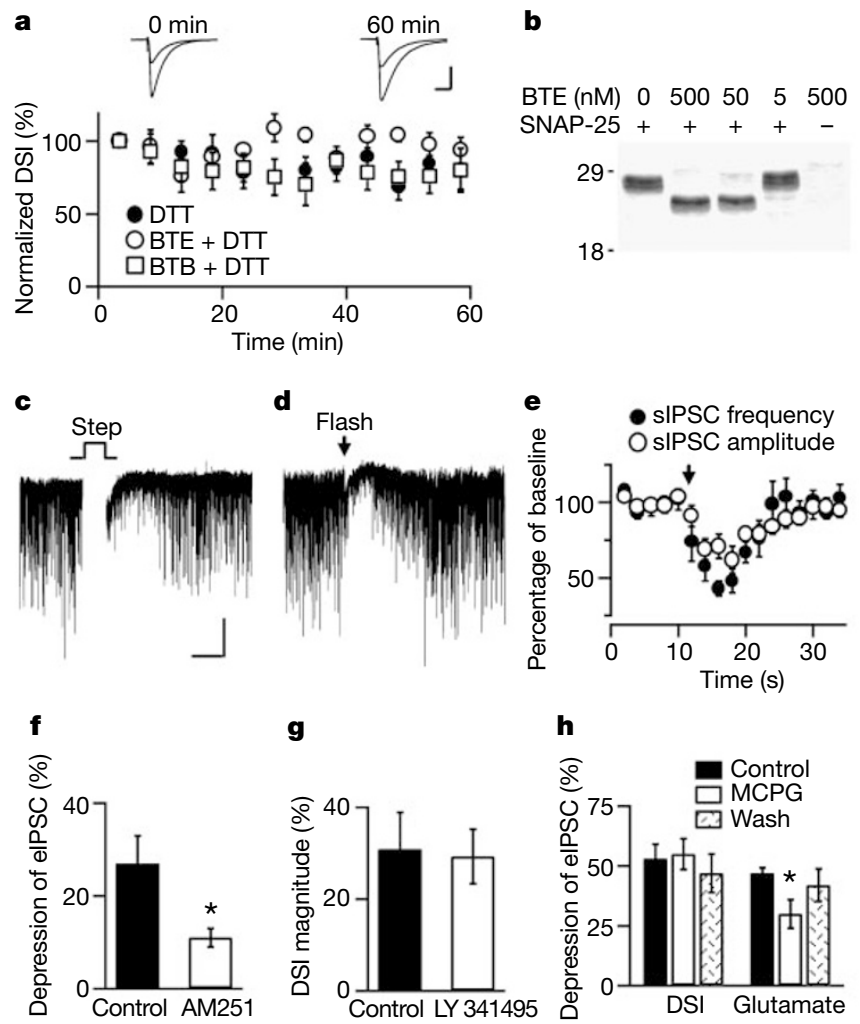

Figure 4 The postsynaptic properties of DSI are consistent with cannabinoids but inconsistent with a classical neurotransmitter. a, Filling pyramidal neurons with BTE $(n=5)$ or BTB $(n=7)(500 \mathrm{nM})$, plus DTT to maximize botulinum toxin activity, had no effect on the stability of DSI compared to DTT $(n=8)$ alone (DSI magnitude at 60 min was $94 \pm 9 \%$ of the average magnitude during first 5 min for BTE and $80 \pm 15 \%$ for BTB; versus $81 \pm 14 \%$ for control). Scale bar: 200 pA, 20 ms. The mean absolute magnitudes of DSI were $52 \pm 3 \%$ (BTE), $45 \pm 3 \%$ (BTB) and $59 \pm 7 \%$ (control). b, 50 nM BTE was sufficient to completely cleave in 60 min all the SNAP-25 diluted in a sample of electrodefilling solution. Relative molecular mass (K) shown on left. c, d, Depolarizing a pyramidal neuron (c) and photolysing caged calcium inside a pyramidal neuron (d) had similar effects on sIPSCs. Scale bar: 50 pA, 10 s. e, Both sIPSC frequency and sIPSC amplitude were transiently depressed by calcium uncaging (frequency $43 \pm 5 \%$ of baseline, amplitude $71 \pm 8 \%$ of baseline, both measured 6 s after flash; $n=8$ cells). f, AM251 $(2 \mu \mathrm{M})$ antagonizes the depression of elPSCs elicited by calcium uncaging $(11 \pm 2 \%$ average depression in AM251 $(n=15)$, versus $27 \pm 6 \%$ average depression in DMSO alone, $(n=16))$. Asterisk, $P<0.01$, $t$-test. $\mathbf{g}$, DSI is normal in slices pre-incubated and recorded in LY341495 (50 $\mu \mathrm{M})(29 \pm 6 \%$ average DSI; $n=10)$ compared to controls (31 $\pm 8 \%$ average DSI; $n=10)$. h, (S)-MCPG (5 mM) does not affect DSI (53 $\pm 6 \%$ average DSI during baseline versus $55 \pm 6 \%$ in (S)-MCPG; $n=6$ ), although it reduces the depression of elPSCs by iontophoresed glutamate in the same recordings ( $47 \pm 2 \%$ average depression during baseline versus $30 \pm 6 \%$ in (S)-MCPG). Asterisk, $P<0.05$, paired $t$-test. grade signal in DSI might be glutamate, although not all studies have found a role for mGluRs in $\mathrm{DSI}^{26}$. Glutamate, or any other classical neurotransmitter, could be released from pyramidal cell somata by either vesicular fusion or by reversal of an electrogenic transporter on the plasma membrane. However, our data imply that neither of these processes can account for the release of the retrograde signal. We therefore re-examined the sensitivity of DSI to mGluR antagonists. The broad-spectrum, high-affinity mGluR antagonist LY341495 (Tocris) did not affect DSI (Fig. 4g). DSI was also resistant to (S)-MCPG (Tocris), although this dose significantly diminished the depression of eIPSCs by glutamate iontophoresed from a second electrode near the recorded cell (Fig. 4h). Our results are inconsistent with the hypothesis that glutamate, or indeed any classical neurotransmitter, is the primary retrograde signal in DSI. Although mGluRs seem not to be required for DSI, our results do not rule out the possibility that mGluRs might have a modulatory function in DSI. Indeed, recent data suggests that mGluR activation can enhance DSI in hippocampal slices (B. E. Alger, personal communication). Differences in experimental preparations could produce different levels of mGluR activation, and could explain the variability in the effect of

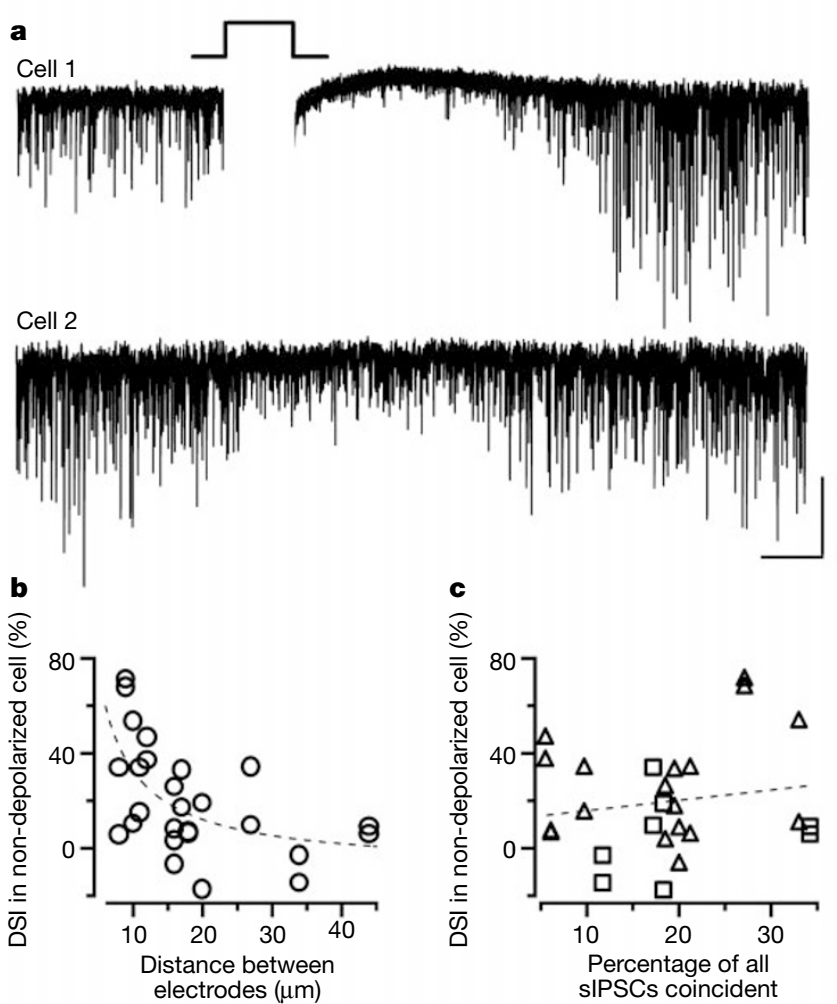

Figure 5 The retrograde signal in DSI can disinhibit nearby neurons. a, Depolarizing one CA1 pyramidal neuron for $5 \mathrm{~s}$ results in a transient suppression of sIPSCs (cell 1). The trace is blanked during the depolarizing step for clarity. A pyramidal cell $9 \mu \mathrm{m}$ away (cell 2) also showed suppression of SIPSCs, beginning about $1 \mathrm{~s}$ after cell 1 was depolarized. Scale bar: 100 pA, 5 s. b, In 13 pyramidal cell pairs, the suppression of sIPSCs in the nondepolarized cell was steeply related to its distance from the depolarized cell, with little propagation beyond $20 \mu \mathrm{m}\left(r^{2}=0.34\right.$, DSI in non-depolarized cell versus 1/distance; $P<0.005$, analysis of variance). The dotted line is a linear fit of DSI in the nondepolarized cell versus $1 /$ distance. $\mathbf{c}$, In the same pairs, suppression of sIPSCs in the nondepolarized cell was not related to the degree of shared presynaptic input, assessed by the percentage of sIPSCs in both cells during the baseline period, which had a coincident event in the other cell $\left(r^{2}=0.03\right.$, DSI in non-depolarized cell versus percentage sIPSCs coincident; not significant, analysis of variance). The dotted line is a linear fit of DSI in the non-depolarized cell versus percentage coincidence. Triangles, close pairs $(<20 \mu \mathrm{m}$ apart); squares, more distant pairs. 
mGluR antagonists.

Endogenous cannabinoids are small diffusible molecules. A third prediction, therefore, is that DSI ought to spread. To test this, we recorded simultaneously in whole-cell mode from pairs of nearby pyramidal neurons. When depolarizing one of these two neurons depressed sIPSCs in that cell, a simultaneous depression of sIPSCs was often observed in the non-depolarized neighbour (Fig. 5a). We made 13 pairs of simultaneous recordings (26 neurons) where both neurons in the paired recording could produce DSI of their own IPSCs, signifying that these neurons were able to produce the retrograde signal and had presynaptic inputs sensitive to the signal. Of these 26 neurons, 10 showed an average suppression of inhibition of at least $20 \%$ immediately after the other neuron in the pair was depolarized. The degree of DSI propagation in these pairs is dependent on distance, with 9 of the 10 propagating pairs separated by $20 \mu \mathrm{m}$ or less (Fig. 5b).

A DSI-like phenomenon in cerebellar Purkinje cells ${ }^{27}$ and cultured myocytes ${ }^{28}$ involves retrograde messengers that locally initiate a signalling cascade in the presynaptic axon terminal; this then propagates the depression through that axon to other synapses. If hippocampal DSI shared this property, then propagation should depend on the degree to which the two postsynaptic cells share some presynaptic inputs, as estimated by the percentage of sIPSCs in the two cells that are coincident. We find that the degree of propagation is uncorrelated with this variable (Fig. 5c), consistent with propagation occurring mainly through a diffusible molecule capable of spreading through a sphere of about $40 \mu \mathrm{m}$ in diameter. This profile is consistent with the properties of the endocannabinoids, whose sphere of diffusion is limited by uptake, and hindered by their lipophilicity ${ }^{8}$.

Here we have placed cannabinoids in a small group of molecules identified as fast retrograde signals in the mature nervous system. Furthermore, our study represents the first identification of a physiological process mediated by endogenous brain cannabinoids. CB1-expressing interneurons are thought to control hippocampal oscillations in the theta and gamma frequency, and a CB1 agonist decreases the power of synchronous oscillations in hippocampal slices $^{11}$, suggesting that endogenous cannabinoids may modulate the synchronous spiking of hippocampal cells. Moreover, as decreasing GABA-mediated inhibition promotes long-term potentiation of glutamatergic synapses ${ }^{29}$, DSI may facilitate learning under conditions of intense hippocampal recruitment. The fact that only strong depolarization can induce DSI means that relevant levels of cannabinoids probably occur in response to particularly intense stimuli. By contrast, exogenous cannabinoids such as marijuana might be predicted to tonically disinhibit the hippocampus, thereby destroying the information contained in the feedback loop of DSI and promoting a noisier, more random pattern of synaptic modification.

Note added in proof: It has come to our attention that two papers showing a role for endocannabinoids are being published in Neuron (Kreitzer, A. C. \& Regehr, W. G. Neuron, 29, 717-727; 2001 and Ohno-Shosaku, T., Maejima, T. \& Kano, M. Neuron, 29, 729-738; 2001).

\section{Methods}

\section{Slice preparation and electrophysiology}

Transverse hippocampal slices $(300 \mu \mathrm{m})$ were obtained from Sprague-Dawley rats (P1630) and maintained in artificial cerebrospinal fluid (ACSF) for at least $1 \mathrm{~h}$ before recording. ACSF contained (in $\mathrm{mM}$ ): $119 \mathrm{NaCl}, 26 \mathrm{NaHCO}_{3}, 10$ glucose, $3 \mathrm{KCl}, 2.5 \mathrm{CaCl}_{2}$, $2 \mathrm{MgSO}_{4}, 1 \mathrm{NaH}_{2} \mathrm{PO}_{4}, 0.005 \mathrm{NBQX}$ (Tocris), $0.002 \mathrm{CPP}$ (Tocris), and was equilibrated with $95 \% \mathrm{O}_{2}$ and $5 \% \mathrm{CO}_{2}$ at $20-22^{\circ} \mathrm{C}$. We added carbachol $(5-10 \mu \mathrm{M}$; RBI) to the ACSF in some experiments (Figs $4 \mathrm{c}-\mathrm{e}$ and 5 ) to increase sIPSC frequency ${ }^{17}$. Recording electrodes were filled with a solution of (in $\mathrm{mM}$ ): $100 \mathrm{CsCH}_{3} \mathrm{SO}_{3}, 60 \mathrm{CsCl}, 5 \mathrm{QX}-314$ chloride, 10 HEPES, 0.2 EGTA, $1 \mathrm{MgCl}_{2}, 1 \mathrm{Mg}$-ATP, and $0.3 \mathrm{Na}_{3} \mathrm{GTP}$ (pH 7.3, 275 mOsM. Synaptic currents were filtered at $2 \mathrm{kHz}$ and collected at $5 \mathrm{kHz}$. When series resistance exceeded $40 \mathrm{M} \Omega$ or input resistance fell below $100 \mathrm{M} \Omega$, experiments were terminated. IPSCs were elicited using bipolar tungsten electrodes in or near CAl stratum pyramidale. DSI tests, performed every $120 \mathrm{~s}$, consisted of 30 stimuli at $0.33 \mathrm{~Hz}$, with depolarization from
$-60 \mathrm{mV}$ to $0 \mathrm{mV}$ for $5 \mathrm{~s}$ after the eighth stimulus. DSI was calculated using the mean of the three eIPSCs just before the depolarization ( amp $_{\text {baseline }}$ ) and the three eIPSCs just after the depolarization $\left(\mathrm{amp}_{\text {test }}\right)$ : DSI $(\%)=100\left(1-\left(\mathrm{amp}_{\text {test }} / \mathrm{amp}_{\text {baseline }}\right)\right)$.

\section{Pharmacology}

Slices were pre-incubated with the drug AM251 (Figs 1a, b and 4f), SR141716 (Fig. 1b) or LY341495 (Fig. 4g) for 45-150 min, and recordings were performed in the presence of the same concentration. Drug-treated slices were interleaved with control slices from the same animal incubated for an equivalent period of time in the same concentration of the solvent (water, dilute $\mathrm{NaOH}$, or DMSO) used to make the drug stock. Overall, about $60 \%$ of cells have significant DSI, with DSI in these cells averaging about $50 \%$ in magnitude; thus, DSI averaged about $30 \%$ in control experiments. For acute applications of drugs (Figs $1 \mathrm{c}-\mathrm{e}$ and $2 \mathrm{a}-\mathrm{d}$ ), cells were discarded immediately if they did not show DSI of at least $30 \%$ during the $10-\mathrm{min}$ baseline period.

\section{Miniature IPSCs}

In experiments examining mIPSCs (Fig. 3c-f) additional $\mathrm{KCl}(2.0-7.5 \mathrm{mM})$ was added to the ACSF at the beginning of the experiment until sIPSC frequency was at least $3 \mathrm{~Hz}$. The ACSF in these experiments included high $\mathrm{Ca}^{2+}\left(7.5 \mathrm{mM}\right.$ total $\left.\mathrm{Ca}^{2+}\right)$ and low magnesium concentration $\left(0.1 \mathrm{mM}\right.$ total $\left.\mathrm{Mg}^{2+}\right)$ to maximize the probability of release, whereas high total divalents served to decrease action-potential initiation. This permitted us, in DSI experiments (Fig. 3e-f), to record both action-potential-dependent sIPSCs and, after addition of TTX, mIPSCs at a frequency of $3-10 \mathrm{~Hz}$ without changing external $\mathrm{KCl}$ concentrations. Phosphate was omitted from the ACSF. In DSI experiments (Fig. 3e-f), cells showing DSI of eIPSCs measuring less than $30 \%$ on the first test (before addition of high external $\mathrm{KCl}$ ) were discarded immediately. Although the cells selected for these experiments (Fig. $3 \mathrm{e}-\mathrm{f}$ ) all had robust DSI in normal ACSF (initial average DSI was $60 \pm 4 \%$ before adding high potassium), elevating external potassium decreased the magnitude of DSI, such that the effect on sIPSC frequency and amplitude was modest, although highly significant. This is consistent with reports that depolarization decreases the efficacy of G-protein-mediated inhibition of voltage-dependent calcium currents $\mathrm{s}^{30}$, and may explain the failure of one study ${ }^{15}$ to find an effect of DSI on $\mathrm{Ca}^{2+}$-dependent mIPSCs, as even higher concentrations of external potassium were used.

A related issue is why we found an increase in the PPR during DSI, whereas other reports ${ }^{15,16}$ have not. In contrast to previous studies, we used a shorter inter-pulse interval ( $55 \mathrm{~ms}$ versus $200 \mathrm{~ms}$ ) to maximize interaction between the two stimuli, and we used a paired $t$-test, which has better statistical resolution than an unpaired comparison. Finally, we note that under optimal conditions DSI might block completely transmission at the subset of terminals expressing CB1 and that, in these cases, a change in the PPR would not be expected.

\section{Botulinum toxins and $\mathrm{Ca}^{2+}$ uncaging}

BTE ( $500 \mathrm{nM}$; a gift from R. Scheller) light chain or BTB ( $500 \mathrm{nM}$; List Biolabs) light chain were dissolved in electrode-filling solution with $5 \mathrm{mM}$ dithiothreitol (DTT). We performed recordings at $31^{\circ} \mathrm{C}$. Cells with DSI of less than $30 \%$ on the first test $(1 \mathrm{~min}$ after break-in) were discarded immediately. For Fig. 4b, recombinant SNAP-25 (a gift from R. Scheller) was diluted to a concentration of $0.2 \mathrm{mg} \mathrm{ml}^{-1}$ in electrode-filling solution, along with $5 \mathrm{mM}$ DTT and $0-500 \mathrm{nM}$ BTE. After incubation for $60 \mathrm{~min}$ at $31^{\circ} \mathrm{C}$, proteins were separated on a $12 \%$ polyacrylamide gel and stained with Coomassie blue. In $\mathrm{Ca}^{2+}$-uncaging experiments (Fig. $4 \mathrm{c}-\mathrm{e}$ ) recording electrodes were filled with a solution containing (in mM): $100 \mathrm{CsCH}_{3} \mathrm{SO}_{3}$, 78 tetraethylammonium chloride, 5 QX-314 chloride, 25 HEPES, $1 \mathrm{MgCl}_{2}, 2 \mathrm{Mg}$-ATP, $0.3 \mathrm{Na}_{3} \mathrm{GTP}, 5$ nitrophenyl-EGTA (Molecular Probes), $4 \mathrm{CsOH}, 2 \mathrm{CaCl}_{2}$. Apamin (100 nM; CalBiochem) was included in the ACSF. Internal tetraethylammonium and external apamin were used to block $\mathrm{Ca}^{2+}$-dependent potassium conductances after uncaging. To uncage nitrophenyl-EGTA, cells were exposed for $800 \mathrm{~ms}$ to ultraviolet light from a $100 \mathrm{~W}$ mercury burner (Olympus) passed through a $25 \%$ neutral density filter.

\section{Paired recordings}

We made 39 pairs of simultaneous recordings from CA1 pyramidal neurons ( 78 neurons); 44 of the $78(56 \%)$ were able to induce DSI of their own IPSCs. This percentage is consistent with the finding that only a subpopulation of interneurons expresses CB1 (ref. 10). Of these 44 neurons, 26 were members of a pair where both neurons were able to produce DSI of their own IPSCs. We analysed this set of recordings to determine under what conditions DSI might spread. The distance between neurons was defined as the distance between the tips of the two recording electrodes. Coincident sIPSCs were defined as events whose peak amplitude occurred within $7 \mathrm{~ms}$ of each other. A 7-ms window was chosen because longer windows did not substantially increase the frequency of calculated coincidence, whereas shorter windows did substantially decrease that frequency; also, average sIPSC rise time sometimes differed by $2-4 \mathrm{~ms}$ between the two cells, probably owing to different series resistances. As DSI of sIPSCs is a decrease in both frequency and amplitude of events, we express DSI here as a function of charge transfer normalized to time, that is, the sum of the areas of all sIPSCs in a period of time divided by the number of seconds in that period. Thus, expressed as a percentage decrease:

$\operatorname{DSI}(\%)=100\left(1-\left(\right.\right.$ charge transfer $_{\text {test }} /$ charge $\left.\left.\operatorname{transfer}_{\text {baseline }}\right)\right)$ where the baseline period was the $20 \mathrm{~s}$ immediately preceding the depolarization, and the test period was the $15 \mathrm{~s}$ immediately after the depolarization. 
1. Matsuda, L. A., Lolait, S. J., Brownstein, M. J., Young, A. C. \& Bonner, T. I. Structure of a cannabinoid receptor and functional expression of the cloned cDNA. Nature 346, 561-564 (1990).

2. Caulfield, M. P. \& Brown, D. A. Cannabinoid receptor agonists inhibit Ca current in NG108-15 neuroblastoma cells via a pertussis toxin-sensitive mechanism. Br. J. Pharmacol. 106, 231-232 (1992)

3. Mackie, K. \& Hille, B. Cannabinoids inhibit N-type calcium channels in neuroblastoma-glioma cells. Proc. Natl Acad. Sci. USA 89, 3825-3829 (1992).

4. Herkenham, M. et al. Cannabinoid receptor localization in brain. Proc. Natl Acad. Sci. USA 87, 1932 1936 (1990).

5. Devane, W. A. et al. Isolation and structure of a brain constituent that binds to the cannabinoid receptor. Science 258, 1946-1949 (1992).

6. Stella, N., Schweitzer, P. \& Piomelli, D. A second endogenous cannabinoid that modulates long-term potentiation. Nature 388, 773-778 (1997).

7. Di Marzo, V. et al. Formation and inactivation of endogenous cannabinoid anandamide in central neurons. Nature 372, 686-691 (1994).

8. Di Marzo, V., Melck, D., Bisogno, T. \& De Petrocellis, L. Endocannabinoids: endogenous cannabinoid receptor ligands with neuromodulatory action. Trends Neurosci. 21, 521-528 (1998).

9. Tsou, K., Mackie, K., Sañudo-Peña, M. C. \& Walker, J. M. Cannabinoid CB1 receptors are localized primarily on cholecystokinin-containing GABAergic interneurons in the rat hippocampal formation Neuroscience 93, 969-975 (1999).

10. Katona, I. et al. Presynaptically located CB1 cannabinoid receptors regulate GABA release from axon terminals of specific hippocampal interneurons. J. Neurosci. 19, 4544-4558 (1999).

11. Hajos, N. et al. Cannabinoids inhibit hippocampal GABAergic transmission and network oscillations. Eur. J. Neurosci. 12, 3239-3249 (2000).

12. Hoffman, A. F. \& Lupica, C. R. Mechanisms of cannabinoid inhibition of GABA(A) synaptic transmission in the hippocampus. J. Neurosci. 20, 2470-2479 (2000)

13. Pitler, T. A. \& Alger, B. E. Postsynaptic spike firing reduces synaptic GABAA responses in hippocampal pyramidal cells. J. Neurosci. 12, 4122-4132 (1992).

14. Lenz, R. A., Wagner, J. J. \& Alger, B. E. N- and L-type calcium channel involvement in depolarization-induced suppression of inhibition in rat hippocampal CA1 cells. J. Physiol. 512, 61-73 (1998).

15. Alger, B. E. et al. Retrograde signalling in depolarization-induced suppression of inhibition in rat hippocampal CA1 cells. J. Physiol. 496, 197-209 (1996).

16. Morishita, W. \& Alger, B. E. Sr2+ supports depolarization-induced suppression of inhibition and provides new evidence for a presynaptic expression mechanism in rat hippocampal slices. J. Physiol. 505, 307-317 (1997).

17. Pitler, T. A. \& Alger, B. E. Depolarization-induced suppression of GABAergic inhibition in rat hippocampal pyramidal cells: $G$ protein involvement in a presynaptic mechanism. Neuron 13, $1447-$ 1455 (1994).

18. Beltramo, M. et al. Functional role of high-affinity anandamide transport, as revealed by selective inhibition. Science 277, 1094-1097 (1997).

19. Piomelli, D. et al. Structural determinants for recognition and translocation by the anandamide transporter. Proc. Natl Acad. Sci. USA 96, 5802-5807 (1999).

20. Binz, T. et al. Proteolysis of SNAP-25 by types E and A botulinal neurotoxins. J. Biol. Chem. 269, 1617 1620 (1994).

21. Leung, S. M., Chen, D., DasGupta, B. R., Whiteheart, S. W. \& Apodaca, G. SNAP-23 requirement for transferrin recycling in Streptolysin-O-permeabilized Madin-Darby canine kidney cells. J. Biol. Chem. 273, 17732-17741 (1998)

22. Weber, T. et al. SNAREpins: minimal machinery for membrane fusion. Cell 92, 759-772 (1998).

23. Lledo, P. M., Zhang, X., Südhof, T. C., Malenka, R. C. \& Nicoll, R. A. Postsynaptic membrane fusion and long-term potentiation. Science 279, 399-403 (1998).

24. Lüscher, C. et al. Role of AMPA receptor cycling in synaptic transmission and plasticity. Neuron 24, 649-658 (1999).

25. Morishita, W., Kirov, S. A. \& Alger, B. E. Evidence for metabotropic glutamate receptor activation in the induction of depolarization-induced suppression of inhibition in hippocampal CAl. J. Neurosci. 18, 4870-4882 (1998)

26. Morishita, W. \& Alger, B. E. Differential effects of the group II mGluR agonist, DCG-IV, on depolarization-induced suppression of inhibition in hippocampal CA1 and CA3 neurons. Hippocampus 10, 261-268 (2000)

27. Vincent, P. \& Marty, A. Neighboring cerebellar Purkinje cells communicate via retrograde inhibition of common presynaptic interneurons. Neuron 11, 885-893 (1993).

28. Cash, S., Zucker, R. S. \& Poo, M. M. Spread of synaptic depression mediated by presynaptic cytoplasmic signaling. Science 272, 998-1001 (1996).

29. Wigström, H. \& Gustafsson, B. Facilitation of hippocampal long-lasting potentiation by GABA antagonists. Acta Physiol. Scand. 125, 159-172 (1985).

30. Brody, D. L. \& Yue, D. T. Relief of G-protein inhibition of calcium channels and short-term synaptic facilitation in cultured hippocampal neurons. J. Neurosci. 20, 889-898 (2000).

\section{Acknowledgements}

We thank J. S. Isaacson and E. Schnell for suggesting a role for cannabinoids in DSI; R. S. Zucker for technical advice in the calcium uncaging experiments; and R. H. Scheller and Y. A. Chen for the gift of recombinant BTE light chain and SNAP-25. We are grateful for the comments on the manuscript contributed by D. S. Bredt, D. R. Copenhagen, R. H. Edwards, M. Frerking, D. Schmitz and M. P. Stryker. R.I.W. is supported by a National Science Foundation Graduate Research Fellowship. R.A.N. is a member of the Keck Center for Integrative Neuroscience and the Silvio Conte Center for Neuroscience Research. R.A.N. was supported by grants from the National Institutes of Health and the Bristol-Myers Squibb Corporation.

Correspondence and requests for materials should be addressed to R.A.N. (e-mail: nicoll@phy.ucsf.edu).

\section{$\mathrm{Ca}^{2+}$ signalling between single L-type $\mathrm{Ca}^{2+}$ channels and ryanodine receptors in heart cells}

\author{
Shi-Qiang Wang ${ }^{\star}$, Long-Sheng Song ${ }^{\star}$, Edward G. Lakatta ${ }^{\star}$ \\ \& Heping Cheng ${ }^{\star} \dagger$
}

* Laboratory of Cardiovascular Sciences, National Institute on Aging, National Institutes of Health, Baltimore, Maryland 21224, USA

$\dagger$ National Laboratory of Biomembrane and Membrane Biotechnology, College of Life Sciences, Peking University, Beijing 100871, China

$\mathrm{Ca}^{2+}$-induced $\mathrm{Ca}^{2+}$ release is a general mechanism that most cells use to amplify $\mathrm{Ca}^{2+}$ signals ${ }^{1-5}$. In heart cells, this mechanism is operated between voltage-gated L-type $\mathrm{Ca}^{2+}$ channels (LCCs) in the plasma membrane and $\mathrm{Ca}^{2+}$ release channels, commonly known as ryanodine receptors, in the sarcoplasmic reticulum ${ }^{3-5}$. The $\mathrm{Ca}^{2+}$ influx through LCCs traverses a cleft of roughly $12 \mathrm{~nm}$ formed by the cell surface and the sarcoplasmic reticulum membrane, and activates adjacent ryanodine receptors to release $\mathrm{Ca}^{2+}$ in the form of $\mathrm{Ca}^{2+}$ sparks ${ }^{6}$. Here we determine the kinetics, fidelity and stoichiometry of coupling between LCCs and ryanodine receptors. We show that the local $\mathrm{Ca}^{2+}$ signal produced by a single opening of an LCC, named a ' $\mathrm{Ca}^{2+}$ sparklet', can trigger about 4-6 ryanodine receptors to generate $\mathrm{C}^{2+}$ spark. The coupling between LCCs and ryanodine receptors is stochastic, as judged by the exponential distribution of the coupling latency. The fraction of sparklets that successfully triggers a spark is less than unity and declines in a use-dependent manner. This optical analysis of singlechannel communication affords a powerful means for elucidating $\mathrm{Ca}^{2+}$-signalling mechanisms at the molecular level.

To visualize the trigger $\mathrm{Ca}^{2+}$ entry through single LCCs in intact rat ventricular myocytes, we used confocal imaging in the line-scan mode to detect the local $\mathrm{Ca}^{2+}$ signal directly beneath the voltageclamped patch membrane ${ }^{7}$ (Fig. 1, inset). The sarcoplasmic reticulum (SR) $\mathrm{Ca}^{2+}$ release was paralysed by $10 \mathrm{mM}$ caffeine and $10 \mu \mathrm{M}$ thapsigargin, an SR $\mathrm{Ca}^{2+}$ pump blocker. When the pipette solution contained $20 \mathrm{mM} \mathrm{Ca}^{2+}$ and $10 \mu \mathrm{M}$ FPL64176 (FPL), an LCC agonist that prolongs channel open time ${ }^{8,9}$, patch depolarization from a holding potential of $-50 \mathrm{mV}$ to test potentials between -40 and $0 \mathrm{mV}$ activated single-channel currents $\left(i_{\mathrm{Ca}}\right)$ in a voltage-dependent manner (Figs 1 and $2 \mathrm{a}$ ). Each open event was accompanied by a discrete minuscule $\mathrm{Ca}^{2+}$ transient, which we named a ' $\mathrm{Ca}^{2+}$ sparklet', in close proximity to the patch membrane. $\mathrm{Ca}^{2+}$ sparklets were resistant to ryanodine $(10 \mu \mathrm{M}, 5 \mathrm{~min}, n=3$ patches $)$, but were completely abolished by the LCC antagonist, nifedipine $(2 \mu \mathrm{M}$, $3 \mathrm{~min}, n=3$ patches). These data indicate that $\mathrm{Ca}^{2+}$ sparklets originate from single LCC openings, and represent the first optical measurement of voltage-gated single-channel activity in intact cells.

Unlike $\mathrm{Ca}^{2+}$ sparks of ryanodine receptor (RyR) origin, which typically last for $\sim 30 \mathrm{~ms}$ (ref. 6 ), $\mathrm{Ca}^{2+}$ sparklets have a variable duration: the onset and termination of $\mathrm{Ca}^{2+}$ sparklets correlated tightly with the open and closure of the channel, respectively (Fig. 1). To characterize sparklet properties in relation to $\mathrm{Ca}^{2+}$ influx, we measured the 'signal mass' of sparklets (that is, the space-time integral of local $\Delta F / F_{0}$ of a sparklet in the line-scan image), and integrated the corresponding unitary $\mathrm{Ca}^{2+}$ influx $\left(q_{\mathrm{Ca}}\right)$. Over the voltage range from $-30 \mathrm{mV}$ to $+10 \mathrm{mV}$, at which LCC openings can be clearly identified, there was a linear correlation between $q_{\mathrm{Ca}}\left(\sim 8,000-100,000 \mathrm{Ca}^{2+}\right.$ ions $)$ and sparklet signal mass (Fig. 2b). Hence, $\mathrm{Ca}^{2+}$ sparklets provide a faithful readout of LCC unitary current.

In heart cells, $\mathrm{Ca}^{2+}$ sparklets from single LCCs are expected to deliver trigger signal into the $12-\mathrm{nm}$ junctional $\mathrm{cleft}^{10,11}$ to activate 